\title{
8. SPYRIDS, ARTOSTROBIIDS, AND CRETACEOUS RADIOLARIANS FROM THE WESTERN PACIFIC, DEEP SEA DRILLING PROJECT LEG $61^{1}$
}

\author{
Patrick de Wever, Université des Sciences et Techniques de Lille, et Equipe de Recherche Associée au C.N.R.S. \\ $\mathrm{n}^{\circ} .764$, SN 5, 59655 Villeneuve d'Ascq, Cedex, France,
}

\begin{abstract}
Investigations have been carried out on Mesozoic and Cenozoic radiolarians of Site 462. Mesozoic species, when identifiable, were used to determine ages. Cenozoic forms occur abundantly from middle Eocene to Pliocene, and show a moderate to good state of preservation. In these series, spyrids and artostrobiids are especially considered with a view to their possible pertinence in establishing biostratigraphic successions.
\end{abstract}

\section{INTRODUCTION}

Only one site was drilled during Leg 61 of the Deep Sea Drilling Project; its location is $7^{\circ} 14.25^{\prime} \mathrm{N}, 165^{\circ}$ $01.83^{\prime} \mathrm{E}$, and water depth is 5181 meters.

Radiolarians are present throughout the sediment cores of Hole 462, although their state of preservation is variable, so that they are sometimes useless for biostratigraphic identification. In Cores 1 to 39 , the radiolarians are abundant and usually well preserved, and they make up the most abundant biogenic component in Cores 23 to 39 . In Cores 41 to 60 , the abundances vary considerably, but generally are less than in the cores above; moreover, the state of preservation is poor at best and, in most cases, so poor that only a few general outlines are visible. Commonly the radiolarians are calcified and the test has been filled and/or replaced by calcite and zeolite.

In material from Hole $462 \mathrm{~A}$, radiolarians are abundant and well preserved in the Neogene cores (Cores 1 and 2). As in Hole 462, they are absent in the Paleogene (Cores 3 to 6) and rare to common, but always poorly preserved, in the Mesozoic sediments.

Aboard the drilling vessel, samples for radiolarian investigation were taken at roughly fixed intervals in the cores. Because most of the sediments are turbidites, it is apparent that the samples are unequally spaced through time. For this reason, samples are much more numerous in some of the time intervals represented. When possible, samples have been taken preferably in red or brown clays.

All the radiolarian samples were processed, aboard the Glomar Challenger, with hydrogen peroxide and Calgon, and with $10 \%$ hydrochloric acid when necessary. The residues were sieved on a $63-\mu \mathrm{m}$ sieve.

\section{SCOPE OF INVESTIGATION}

A survey of the occurrence of Cenozoic forms of known stratigraphic utility is given in another chapter of this volume (see Sanfilippo et al.). The part of the present chapter dealing with Cenozoic radiolarians surveys

\footnotetext{
${ }^{1}$ Initial Reports of the Deep Sea Drilling Project, Volume 61.
}

the spyrids and artostrobiids to determine whether some forms not previously used stratigraphically show promise of stratigraphic utility and whether some others are clearly useless.

Mesozoic radiolarians have been investigated for all the samples in which they were identifiable, in order to determine ages.

\section{CENOZOIC RADIOLARIANS}

\section{Results}

For the Cenozoic sequence of Hole 462, occurrences and abundances of spyrids and artostrobiids have been tabulated in stratigraphic order, as far as possible (Table 1). Species are numbered consecutively, and these numbers are included in the species list.

In the tabulation, as in the paper by Sanfilippo et al. (this volume), indications of abundance and preservation and five columns for reworking are given. The existence of turbidites limits the usefulness of this arrangement, so in order to have a stratigraphic order of species less influenced by the reworking, two other tables ( 2 and 3 ) are presented, taking into account only the samples with less than $1 \%$ of the specimens reworked, accordingly to the percentages shown in Table 1 .

Letters and symbols in the tables are defined as follows:

$\begin{array}{lll}\text { Preservation: } & \text { G } & \begin{array}{l}\text { Good } \\ \text { Moderate }\end{array} \\ & \text { P } & \begin{array}{l}\text { Poor } \\ \text { Abundance: }\end{array} \\ & \text { A } & \begin{array}{l}\text { Abundant (more than } 500 \\ \text { specimens counted) }\end{array} \\ & \text { C } & \begin{array}{l}\text { Common (51 to 500 } \\ \text { specimens counted) }\end{array} \\ & \text { F } & \begin{array}{l}\text { Few (11 to 50 specimens } \\ \text { counted) }\end{array} \\ & \text { R } & \begin{array}{l}\text { Rare (4 to 10 specimens } \\ \text { counted) }\end{array} \\ & + & \begin{array}{l}\text { Very rare (less than 4 speci- } \\ \text { mens per slide) }\end{array} \\ & \text { ? } & \text { Uncertain identification }\end{array}$

From Table 2, it appears that several species are definitely useless for stratigraphic purposes; these in- 
Table 1. Cenozoic radiolarians, Hole 462. (See text for explanation.)

\begin{tabular}{|c|c|c|c|c|c|c|c|c|c|c|c|c|c|c|c|c|c|c|c|c|c|c|}
\hline \multirow[b]{2}{*}{ Chronostratigraphy } & \multirow[b]{2}{*}{$\begin{array}{c}\text { Sample } \\
\text { (interval } \\
\text { in } \mathrm{cm} \text { ) }\end{array}$} & & \multirow{2}{*}{ 点 } & \multicolumn{4}{|c|}{$\begin{array}{l}\text { Percentage of } \\
\text { Specimens } \\
\text { Reworked }\end{array}$} & \multirow{2}{*}{\multicolumn{2}{|c|}{ 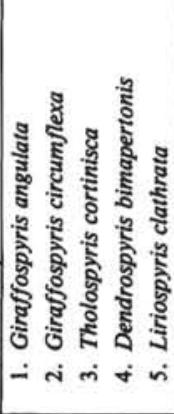 }} & \multirow{2}{*}{\multicolumn{3}{|c|}{ 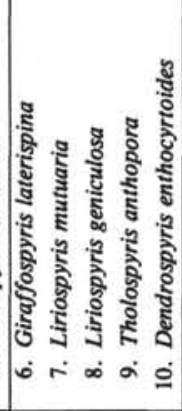 }} & \multirow{2}{*}{\multicolumn{3}{|c|}{ 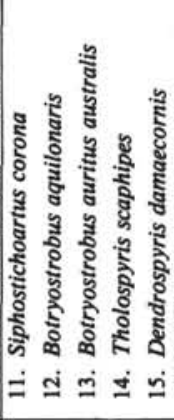 }} & \multirow{2}{*}{\multicolumn{3}{|c|}{ 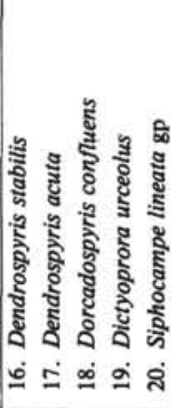 }} & \multirow{2}{*}{\multicolumn{2}{|c|}{ 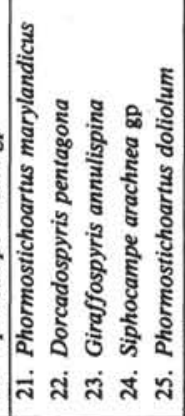 }} & \multirow{2}{*}{\multicolumn{2}{|c|}{ 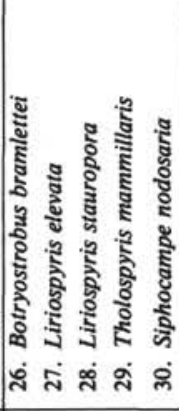 }} \\
\hline & & & & 递 & & 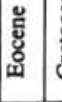 & & & & & & & & & & & & & & & & \\
\hline Pleistocene & $2, \mathrm{CC}$ & & $\mathbf{M}$ & 1 & 0 & 0 & & $F \quad R$ & $++t$ & $+R+1$ & R R & $\mathbf{R}+$ & $++R$ & & & & & & & & & \\
\hline Pliocene & $\begin{array}{l}3, \mathrm{CC} \\
4, \mathrm{CC}\end{array}$ & & \begin{tabular}{l|l}
$\mathbf{M}$ \\
$\mathbf{M}$
\end{tabular} & & $\begin{array}{l}0 \\
0\end{array}$ & $\begin{array}{l}0 \\
0\end{array}$ & & & $\begin{array}{l}+\mathrm{F} \\
\mathrm{F}+\end{array}$ & $\mathrm{F} \underset{\mathrm{F}}{+} \mathrm{R}$ & $\begin{array}{l}++ \\
++\end{array}$ & $\begin{array}{r}+\quad R \\
+\quad+ \\
\end{array}$ & $\mathrm{F}^{+}$ & $\begin{array}{ll}\mathrm{R} & \mathrm{F} \\
\mathrm{F} & \mathrm{F} \\
\end{array}$ & & $\begin{array}{l}\mathbf{R}+\mathbf{R} \\
+\quad \mathbf{R} \\
\end{array}$ & $\begin{array}{l}\mathbf{R} \quad \mathbf{R} \\
\mathbf{R}\end{array}$ & & ${ }^{+} \mathrm{F}$ & +++ & + & \\
\hline $\begin{array}{l}\text { Upper Miocene- } \\
\text { Pliocene }\end{array}$ & $\begin{array}{l}5, \mathrm{CC} \\
6-1,56-58 \\
6-3,65-67 \\
7-1,117-119 \\
7-3,119-121 \\
8-1,107-109 \\
8-3,118-120 \\
\end{array}$ & & \begin{tabular}{|l|}
$\mathrm{M}$ \\
$\mathrm{M}$ \\
$\mathrm{M}$ \\
$\mathrm{M}$ \\
$\mathrm{M}$ \\
$\mathrm{M}$ \\
$\mathrm{G}$ \\
\end{tabular} & $\begin{array}{l}5 \\
5 \\
5 \\
1 \\
5 \\
1\end{array}$ & $\begin{array}{l}0 \\
0 \\
0 \\
1 \\
0 \\
0 \\
0 \\
\end{array}$ & $\begin{array}{l}0 \\
1 \\
1 \\
1 \\
1 \\
1 \\
0 \\
\end{array}$ & & $\begin{array}{ll}R & \\
+ & R \\
R & F \\
& F \\
& R \\
& F \\
F & \text { C } \\
\end{array}$ & 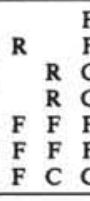 & \begin{tabular}{l|ll}
$\mathbf{F}$ & $R$ & $F$ \\
$F$ & + & $F$ \\
$C$ & $F$ & $C$ \\
$C$ & $R$ & $C$ \\
$F$ & $F$ & $C$ \\
$F$ & $R$ & $C$ \\
$C$ & $F$ & $C$ \\
\end{tabular} & $\begin{array}{ll}\text { F } & \\
\text { C } & F \\
\text { C } & C \\
\text { C } & C \\
\text { C } & C \\
\text { F } & \text { F } \\
\text { F } & \text { R } \\
\end{array}$ & $\begin{array}{ll} & R \\
F & \\
C & R \\
C & R \\
C & \\
F & \\
R & \\
\end{array}$ & $\begin{array}{l}\text { R }+R \\
\text { F R } \\
++ \\
\text { F } \\
\text { C }+\end{array}$ & 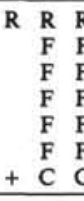 & & $\begin{array}{ll}\mathbf{R} & + \\
& \mathbf{R} \\
& \mathrm{R} \\
& \mathrm{F} \\
& \mathrm{R} \\
& \mathrm{F} \\
& \mathrm{F} \\
\end{array}$ & $\begin{array}{ll}+ & \\
R & \\
R & \\
\text { F } & \text { F } \\
R & F \\
F & R \\
F & F \\
\end{array}$ & $\begin{array}{l}\mathrm{F} \\
\mathrm{F} \\
\mathrm{F} \\
\mathrm{F} \\
\mathrm{F} \\
\mathrm{C} \\
\mathrm{F} \\
\end{array}$ & \begin{tabular}{|ll} 
& F \\
F & F \\
R & R \\
R & C \\
F & C \\
F & C \\
C & F \\
\end{tabular} & $\begin{array}{r}+ \\
+\quad+ \\
+ \\
\\
\\
\end{array}$ & $\begin{array}{rrr}+ & + \\
& \mathrm{R} \\
& \mathrm{R} \\
\mathrm{C} & \mathrm{C} \\
\mathrm{C} & \mathrm{C} & \\
\mathrm{F} & \mathrm{F} & \\
\mathrm{R} & \mathrm{F} & \mathrm{F} \\
\end{array}$ & $\begin{array}{ll}R & + \\
C & ? \\
C & R \\
C & \\
R & \\
\text { R }\end{array}$ \\
\hline $\begin{array}{l}\text { Middle-Upper } \\
\text { Miocene }\end{array}$ & $\begin{array}{l}10-2,67-69 \\
10-5,50-52 \\
11-1,147-149 \\
11-2,77-79 \\
12-1,39-41 \\
12-5,23-25\end{array}$ & & \begin{tabular}{l|l|}
$\mathrm{M}$ & \\
$\mathrm{M}$ & \\
$\mathrm{M}$ & \\
$\mathrm{M}$ & \\
$\mathrm{P}$ & \\
$\mathrm{P}$ & \\
\end{tabular} & & $\begin{array}{l}0 \\
0\end{array}$ & $\begin{array}{l}0 \\
1\end{array}$ & & $\begin{array}{ll}\mathrm{F} & \mathrm{C} \\
\mathrm{F} & \mathrm{F} \\
\mathrm{F} & \mathrm{C} \\
+ & \mathrm{C} \\
+ & \\
\end{array}$ & $\begin{array}{lll}\text { F } & \text { R } & F \\
+ & F & F \\
F & & F \\
C & F & F \\
& + & F \\
+ & F & C \\
\end{array}$ & \begin{tabular}{l|ll}
$F$ & $F$ & $C$ \\
$F$ & $F$ & $F$ \\
$F$ & & $C$ \\
$F$ & + & $C$ \\
$F$ & & $C$ \\
$C$ & & $C$
\end{tabular} & $\begin{array}{ll}\mathrm{C} & \mathrm{F} \\
\mathrm{C} & \mathrm{F} \\
\mathrm{C} & \mathrm{F} \\
\mathrm{C} & \mathrm{F} \\
\mathrm{C} & \mathrm{C} \\
\mathrm{C} & \mathrm{C} \\
\end{array}$ & $\begin{array}{l}\mathbf{F} \\
\mathrm{F} \\
\mathrm{F} \\
\mathrm{F} \\
\mathrm{C} \\
\mathrm{C} \\
\end{array}$ & 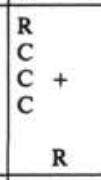 & $\begin{array}{ll}\text { R } & \text { F } \\
\text { F } & \text { F } \\
\text { C } & C \\
\text { F } & C \\
& \text { I } \\
\text { F } & \text { F } \\
\end{array}$ & & $\begin{array}{l}\text { F } \\
\text { C } \\
\text { F }\end{array}$ & $\begin{array}{ll} & R \\
F & R \\
C & F \\
F & R\end{array}$ & & $\begin{array}{|ll|}\mathrm{R} & \mathrm{C} \\
\mathrm{C} & \mathrm{C} \\
\mathrm{C} & \mathrm{C} \\
\mathrm{C} & \mathrm{C} \\
& \mathrm{F} \\
& \mathrm{C} \\
\end{array}$ & $\begin{array}{l}\mathrm{F} \\
\mathrm{F} \\
+\end{array}$ & \begin{tabular}{lll} 
& + & \\
& & \\
$\mathrm{R}$ & \multicolumn{1}{c}{} \\
& & \\
& $\mathrm{C}$ & $\mathrm{F}$ \\
& + & $\mathrm{F}$ \\
$\mathrm{F}$ & $\mathrm{F}$ & $\mathrm{C}$ \\
\end{tabular} & $\begin{array}{l}\mathrm{C} \\
\mathrm{C}+ \\
\mathrm{C} \\
\mathrm{R} \\
\mathrm{R} \\
\mathrm{F} \\
\mathrm{C} \\
\end{array}$ \\
\hline $\begin{array}{l}\text { Middle } \\
\text { Miocene }\end{array}$ & $\begin{array}{l}13-2,88-90 \\
13-5,88-90 \\
14-2,86-88 \\
14-5,109-111 \\
15, \mathrm{CC}\end{array}$ & & \begin{tabular}{|l|}
$\mathrm{M}$ \\
$\mathrm{M}$ \\
$\mathrm{M}$ \\
$\mathrm{M}$ \\
$\mathrm{M}$ \\
\end{tabular} & & $\begin{array}{l}1 \\
0 \\
1 \\
0 \\
\end{array}$ & $\begin{array}{l}1 \\
1 \\
1 \\
1 \\
1 \\
\end{array}$ & & $\begin{array}{r}0 \\
R_{+}^{+} \\
\end{array}$ & 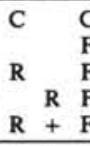 & \begin{tabular}{l|l}
$C$ & \\
$F$ & $R$ \\
$F$ & $C$ \\
$F$ & $C$ \\
$F$ & $C$ \\
$F$ & $C$ \\
\end{tabular} & $\begin{array}{ll}\mathrm{C} & \mathrm{C} \\
\mathrm{A} & \mathrm{C} \\
\mathrm{C} & \mathrm{C} \\
\mathrm{C} & \mathrm{C} \\
\mathrm{C} & \mathrm{F} \\
\end{array}$ & $\begin{array}{l}\mathrm{C} \\
\mathrm{C} \\
\mathrm{C} \\
\mathrm{C} \\
\mathrm{F} \\
\end{array}$ & $\begin{array}{l}++ \\
\mathrm{R} \\
+ \\
+\end{array}$ & $\begin{array}{r}\mathrm{CF} \\
\mathrm{FF} \\
+\mathrm{FF} \\
\mathrm{FF} \\
+\mathrm{R} \\
\end{array}$ & & $\begin{array}{l}\mathrm{C} \\
\mathrm{F} \\
\mathrm{C} \\
\mathrm{R} \\
+\end{array}$ & $\begin{array}{ll}\mathrm{C} & \\
\mathrm{F} & \mathrm{R} \\
\mathrm{C} & + \\
\mathrm{R} & \mathrm{F} \\
+ & \\
\end{array}$ & & \begin{tabular}{|ll}
$\mathrm{C}$ & $\mathrm{C}$ \\
$\mathrm{C}$ & $\mathrm{C}$ \\
& $\mathrm{C}$ \\
$\mathrm{C}$ & $\mathrm{C}$ \\
$\mathrm{F}$ & $\mathrm{F}$ \\
\end{tabular} & + & $\begin{array}{l}\mathrm{F} \\
\mathrm{C} \\
\mathrm{F} \\
\mathrm{C}\end{array}$ & $\begin{array}{l}\text { A } \\
\text { A } \\
\text { C } \\
\text { C } \\
\text { C }\end{array}$ \\
\hline $\begin{array}{l}\text { Lower } \\
\text { Miocene }\end{array}$ & $\begin{array}{l}16-2,68-70 \\
17-2,104-106 \\
17-3,55-57 \\
18-3,85-87 \\
18-5,74-76 \\
\end{array}$ & & \begin{tabular}{l|l}
$\mathrm{P}$ & \\
$\mathrm{M}$ & \\
$\mathrm{M}$ & \\
$\mathrm{P}$ & \\
$\mathrm{P}$ & \\
\end{tabular} & & 1 & \begin{tabular}{l|l}
1 & \\
1 & \\
0 & \\
1 & \\
1 & \\
\end{tabular} & & $\mathbf{R}$ & $\begin{array}{rr}+ & + \\
& R \\
& R \\
R & R \\
R\end{array}$ & 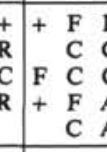 & $\begin{array}{ll}\text { F } & \text { F } \\
\text { C } & \text { C } \\
\text { C } & \text { C } \\
\text { A } & \text { C } \\
\text { A } & \text { C } \\
\end{array}$ & $\begin{array}{l}\mathbf{F} \\
\mathrm{C} \\
\mathrm{C} \\
\mathrm{C} \\
\mathrm{C}\end{array}$ & & $\begin{array}{ll}+ & \\
\mathrm{R} & \mathrm{R} \\
\mathrm{F} & \mathrm{F} \\
\mathrm{R} & \mathrm{R}\end{array}$ & & $\begin{array}{r}\mathbf{R} \\
+\mathrm{F} \\
\quad \mathrm{F} \\
+\end{array}$ & $\begin{array}{ll}\mathrm{F} & \\
\mathrm{F} & \mathrm{R} \\
+ \\
+ \\
\end{array}$ & & $\begin{array}{ll}++ & \text { F } \\
\text { R } & \text { C } \\
\text { C } & \text { C } \\
\text { F } & \text { C } \\
\text { F } & \text { A } \\
\end{array}$ & & + & $\begin{array}{l}\text { C } \\
\text { C } \\
\text { C } \\
\text { A } \\
\text { C }\end{array}$ \\
\hline $\begin{array}{l}\text { Upper } \\
\text { Oligocene }\end{array}$ & $\begin{array}{l}19-2,142-144 \\
91-6,23-25 \\
20-4,25-27 \\
21-1,35-37 \\
22-3,106-108 \\
23-1,131-133 \\
23-4,67-69 \\
24-2,81-83 \\
25-1,142-144 \\
25-4,141-143 \\
\end{array}$ & & \begin{tabular}{l|l}
$\mathrm{P}$ & 0 \\
$\mathrm{M}$ & \\
$\mathrm{M}$ & \\
$\mathrm{M}$ & \\
$\mathrm{M}$ & \\
$\mathrm{M}$ & \\
$\mathrm{M}$ & \\
$\mathrm{M}$ & \\
$\mathrm{M}$ & \\
$\mathrm{M}$ & \\
\end{tabular} & 0 & & \begin{tabular}{r|r}
20 & 0 \\
0 & \\
5 & \\
10 & \\
5 & \\
20 & \\
20 &
\end{tabular} & 0 & $\begin{array}{l}\mathbf{R} \\
+\end{array}$ & $\begin{array}{rr} & F \\
+ & R F \\
& F \\
R & F \\
& F \\
& F \\
& F \\
& F \\
R \quad F\end{array}$ & 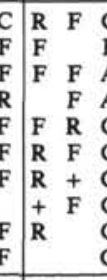 & $\begin{array}{ll}\text { C } & \text { C } \\
\text { F } & \\
\text { A } & \text { C } \\
\text { A } & \text { C } \\
\text { C } & \text { C } \\
\text { C } & \text { R } \\
\text { C } & \text { F } \\
\text { C } & \\
\text { C } & \\
\text { C } & +\end{array}$ & $\begin{array}{l}\mathrm{C} \\
\mathrm{C} \\
\mathrm{C} \\
\mathrm{C} \\
\mathrm{R} \\
\mathrm{F}\end{array}$ & R & 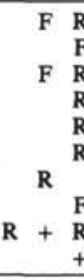 & $\begin{array}{l}\mathrm{R} \\
\mathrm{F} \\
\mathrm{R} \\
\mathrm{R} \\
\mathrm{R} \\
\mathrm{R} \\
\mathrm{F} \\
\mathrm{R} \\
+ \\
\end{array}$ & $\begin{array}{r}+\mathrm{C} \\
\mathrm{C} \\
\mathrm{F} \\
\mathrm{C} \\
\mathrm{F} \\
\mathrm{F} \\
+\quad \mathrm{C} \\
\mathrm{F} \\
\mathrm{F} \\
\mathrm{C}\end{array}$ & $\begin{array}{ll}C & C \\
C & \\
F & \\
C & F \\
F & \\
F & F \\
C & \\
F & \\
F & \\
C & \\
\end{array}$ & \begin{tabular}{l|l}
$\mathrm{F}$ \\
$\mathrm{F}$ \\
$\mathrm{F}$ \\
$\mathrm{C}$ \\
$\mathrm{F}$ \\
\\
$\mathrm{R}$ \\
$\mathrm{F}$ \\
$\mathrm{F}$ \\
$\mathrm{C}$
\end{tabular} & $\begin{array}{ll}\mathrm{C} & \mathrm{C} \\
\mathrm{R} & \mathrm{C} \\
\mathrm{R} & \mathrm{F} \\
\mathrm{C} & \mathrm{C} \\
\mathrm{F} & \mathrm{F} \\
\mathrm{F} & \mathrm{R} \\
\mathrm{R} & \\
\mathrm{C} & \mathrm{R} \\
\mathrm{C} & \mathrm{F} \\
\mathrm{C} & \mathrm{F} \\
\end{array}$ & + & F R & $\begin{array}{l}\text { C R } \\
\text { F } \\
\text { A } \\
\text { C } \\
\text { C } \\
\text { C }+ \\
\text { C } \\
\text { F F } \\
\text { F }+ \\
\text { F }+\end{array}$ \\
\hline $\begin{array}{l}\text { Upper Eocene- } \\
\text { Lower Oligocene }\end{array}$ & $\begin{array}{l}26-2,62-64 \\
26-4,33-35 \\
27-1,116-118 \\
27-4,47-49 \\
28-2,109-111 \\
28-6,99-101 \\
29-1,109-111 \\
29-5,70-72 \\
30-3,14-16 \\
32-4,60-62 \\
31-2,23-25 \\
33-1,77-79 \\
33-2,24-26 \\
\end{array}$ & & \begin{tabular}{l|l|}
$\mathrm{P}$ & \\
$\mathrm{P}$ & \\
$\mathrm{M}$ & \\
$\mathrm{M}$ & \\
$\mathrm{M}$ & \\
$\mathrm{M}$ & \\
$\mathrm{M}$ & \\
$\mathrm{M}$ & \\
$\mathrm{M}$ & \\
$\mathrm{M}$ & \\
$\mathrm{M}$ & \\
\end{tabular} & & & $\begin{array}{l}1 \\
1\end{array}$ & & F & $\begin{array}{r}\mathrm{R} \\
\mathrm{C} \\
+\quad \mathrm{F} \\
\mathrm{C} \\
\mathrm{F} \\
\mathrm{C} \\
+\mathrm{R} \\
\mathrm{C} \\
\mathrm{R} \\
\mathrm{F} \\
+\mathrm{C} \\
\mathrm{C} \\
\mathrm{C} \\
+\mathrm{C} \\
\end{array}$ & 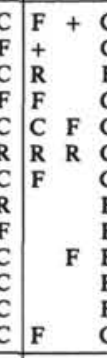 & $\begin{array}{ll}\text { C } & \text { F } \\
\text { C } & \text { F } \\
\text { F } & \\
\text { C } & + \\
\text { C } & + \\
\text { C } & \text { C } \\
\text { C } & \text { R } \\
\text { F } & \text { R } \\
\text { F } & \\
\text { F } \\
\text { F } \\
\text { F } & \text { R } \\
\text { C } & \\
\end{array}$ & $\begin{array}{l}\mathbf{F} \\
\mathbf{F} \\
+ \\
+ \\
\mathbf{C} \\
\mathrm{R} \\
\mathrm{R}\end{array}$ & & $+{ }^{R}$ & $\begin{array}{l}\mathbf{R} \\
\mathbf{R} \\
\mathbf{R}\end{array}$ & $\begin{array}{l}\text { F } \\
+ \\
\text { F } \\
\text { R } \\
+ \\
+ \\
\text { F } \\
\text { F } \\
\text { F } \\
\text { C } \\
\text { R } \\
+\end{array}$ & 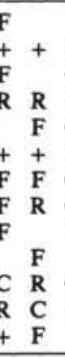 & \begin{tabular}{l|l|} 
F & \\
$C$ & \\
$C$ & \\
$C$ & \\
$F$ & \\
$C$ & \\
$C$ & \\
$F$ & \\
$C$ & \\
$F$ & \\
\end{tabular} & \begin{tabular}{|ll} 
& C \\
+ & R \\
F & R \\
F & C \\
+ & F \\
C & R \\
F & R \\
F & F \\
& F \\
& F \\
& F \\
& C \\
& F \\
\end{tabular} & + & & $\begin{array}{ll}\text { C } \\
\text { C } \\
+ \\
\text { F } \\
\text { C } \\
\text { C } & \text { R } \\
\text { F } & \text { R } \\
\text { C } & \\
\text { C } & \\
\text { F } & \text { C } \\
\text { F } & \\
\text { C } & \\
\text { F } & \\
\end{array}$ \\
\hline Upper Eocene & $\begin{array}{l}34, \mathrm{CC} \\
35-1,15-17 \\
36-1,77-79 \\
36-2,103-105 \\
37-2,42-44 \\
37-5,65-67\end{array}$ & & \begin{tabular}{l|l} 
& \\
$M$ & \\
$M$ & \\
$M$ & \\
$M$ & \\
$M$ &
\end{tabular} & & & \begin{tabular}{r|}
50 \\
1 \\
1 \\
10 \\
30 \\
\end{tabular} & & & $\begin{array}{r}F \\
F \\
+\quad C \\
F \\
+C \\
R C\end{array}$ & 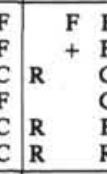 & $\begin{array}{ll}\mathbf{F} & \mathrm{F} \\
\mathrm{F} & + \\
\mathrm{C} & \\
\mathrm{C} & \mathrm{F} \\
\mathrm{F} & + \\
\mathrm{R} & +\end{array}$ & 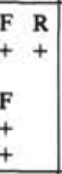 & & & + & $\begin{array}{l}+ \\
\text { F } \\
\text { C } \\
\text { C } \\
\text { F }\end{array}$ & $\begin{array}{ll}+ & C \\
F & \\
C & R \\
F & F \\
R\end{array}$ & \begin{tabular}{l|l}
$\mathrm{C}$ \\
$\mathrm{C}$ \\
\\
$\mathrm{F}$
\end{tabular} & $\begin{array}{rl}R & R \\
& + \\
& +\end{array}$ & + & & $\begin{array}{l}\mathrm{F} \\
\mathrm{F} \\
+ \\
\mathrm{C} \\
\mathrm{F} \\
\mathrm{R}\end{array}$ \\
\hline Middle Eocene & $\begin{array}{l}38-2,100-102 \\
38-5,66-68 \\
39-2,104-106 \\
39-5,9-11 \\
41, C C\end{array}$ & & \begin{tabular}{l|l}
$\mathbf{M}$ & \\
$\mathbf{M}$ & \\
$\mathbf{M}$ & \\
$\mathbf{M}$ &
\end{tabular} & & & \begin{tabular}{c|}
10 \\
20 \\
$?$ \\
$?$
\end{tabular} & & & $\begin{array}{r}\mathrm{C} \\
\mathrm{C} \\
+\mathrm{C} \\
\mathrm{R} \\
\mathrm{C}\end{array}$ & 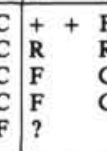 & $\begin{array}{ll}\mathrm{F} & \\
\mathrm{R} & \mathrm{R} \\
\mathrm{C} & \\
\mathrm{C} & \end{array}$ & R & & & & $\begin{array}{l}+ \\
+ \\
\mathrm{F} \\
\mathrm{F} \\
\mathrm{F}\end{array}$ & $\begin{array}{l}+\mathrm{F} \\
+ \\
\mathrm{F} \\
\mathrm{F} \\
\mathrm{F}\end{array}$ & & & & & \\
\hline
\end{tabular}


Table 1. (Continued).

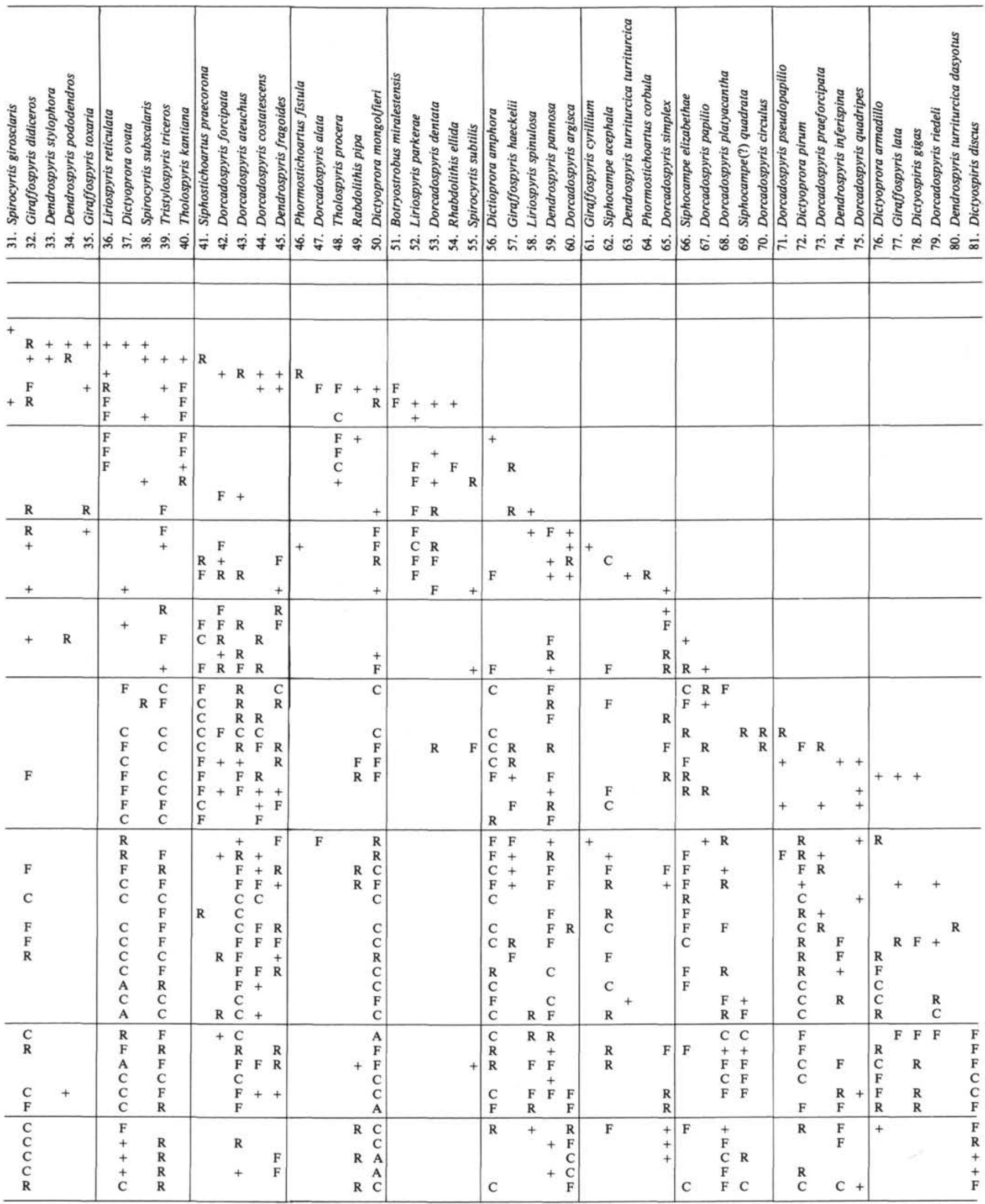


clude Tholospyris cortinisca, Liriospyris mutuaria, Tholospyris anthopora, Dendrospyris damaecornis, Dorcadospyris confluens, Dictyoprora urceolus, Siphostichoartus lineata group, Dorcadospyris pentagona, and, to a lesser extent, Phormostichoartus marylandicus.

Some other species, having short ranges and distinct upper and lower limits, seem to be promising. These are, for example, Liriospyris parkerae (middle Miocene), L. stauropora (middle Miocene to upper part of lower Miocene), Siphostichoartus praecorona (Oligocene to lower Miocene), Siphostichoartus elizabethae (upper Eocene to Oligocene), and Dictyoprora armadillo (Eocene). Even if one section is not sufficiently significant, we may note that first occurrences of some species correspond to last occurrences of others: Dictyoprora ovata and Dorcadospyris simplex disappear (Sample 462-14-5, 109-111 cm with the first occurrence of $L$. parkerae, and the same pertains to Dendrospyris inferispina, Dictyoprora gigas, Dorcadospyris riedeli, and Dorcadospyris praeforcipata (Sample 462-29-1, 109-11 $\mathrm{cm})$.

In order to avoid a long discussion - which might be confusing and which could be taken more seriously than I would like, because of the limited number of samples taken in account-Tables 2 and 3 present the different species arranged in stratigraphic order corresponding to the tops (last occurrences, Table 2) and bottoms (first occurrences, Table 3 ). Tables 2 and 3 permit easy comparison of the tops and bottoms for different species.

\section{Comparisons with Previous Work}

Only a few authors have previously made detailed surveys of spyrids and artostrobiids. The most detailed work on spyrids is that by Goll $(1968,1969,1972)$, and Nigrini (1977) has recently reviewed tropical artostrobiids, so it may be interesting to see how our results fit with theirs.

The ranges of artostrobiids surveyed here correlate well with those established by Nigrini (1977, p. 247). Several remarks, however, seem worthwhile: (1) Siphostichoartus praecorona seems to have a longer range at Hole 462, occurring in some sediments older than those in which it was found by Nigrini (1977). The same is true of Botryostrobus auritus-australis, which occurs rarely from upper Oligocene to Pliocene. (2) Siphocampe acephala, S. elizabethae, Dictyoprora ovata, D. amphora, and $D$. mongolfieri have a few occurrences younger than those given by Nigrini (1977), but they may result from reworking. (3) Reworking could be the reason also for the range of Dictyoprora urceolus, but this is not likely, because the occurrences of this form are very frequent.

Comparing the results presented here with Goll's results, the following remarks are pertinent:

1) For my results to be comparable with Goll's, it is necessary to add the ranges of species from his 1968-1969 papers with those of 1972; taken singly, his ranges seem too restricted.

2) Leaving aside the younger occurrences of several species here, to avoid setting up differences where perhaps they are only a consequence of reworking, sev- eral differences between my ranges and Goll's are apparent. The main differences are as follows: (A) Giraffospyris angulata occurs in Hole 462 from lower Miocene to Pleistocene (upper Miocene-Pliocene, according to Goll). (B) Liriospyris mutuaria occurs from upper Eocene to Pleistocene (lower Miocene to Pliocene, according to Goll). (C) Tholospyris cortinisca occurs from upper Eocene to Pleistocene (middle Miocene to Pliocene, according to Goll). (D) T. scaphipes occurs from upper Oligocene to Pliocene (middle Miocene to Pliocene, according to Goll). (E) T. mammillaris occurs from upper Eocene-lower Oligocene to upper Miocene-Pliocene (upper Oligocene to Pliocene, according to Goll). (F) Giraffospyris circumflexa occurs from upper Oligocene to Pleistocene (middle Miocene to Pliocene, according to Goll).

\section{LIST OF CENOZOIC SPECIES}

The purpose of this listing is to provide bibliographic references to the taxa mentioned in this report and in its accompanying tables. When the published literature contains several different concepts of the limits of a species, the reference cited conforms to the concept as applied here. For each species, this list gives at least one recent reference containing appropriate species descriptions and illustrations, thereby enabling the reader to determine the criteria which have been applied in identifying the taxa in this report.

Botryostrobus aquilonaris (Bailey). Nigrini, 1977, p. 246, pl. 1, fig. 1. Table 1, number 12; Tables 2, 3, number 1'.

Botryostrobus auritus-australis (Ehrenberg) group. Nigrini, 1977, p. 246, pl. 1, figs. 2-5. Table 1, number 13; Tables 2, 3, number $5^{\prime}$. Botryostrobus bramlettei (Campbell and Clark). Nigrini, 1977, p. 248 , pl. 1 , figs. 7,8 . Table 1 , number 26 ; Tables 2 , 3, number $24^{\prime}$. Botryostrobus miralestensis (Campbell and Clark). Nigrini, 1977, p. 249 , pl. 1, fig. 9. Table 1, number 51; Tables 2, 3, number 39'.

Ceratospyris articulata Ehrenberg, 1873, p. 218. Sanfilippo and Riedel, 1973 , p. 526 , pl. 15 , figs. $1-3$; pl. 31 , figs. $8,9$.

Dendrospyris acuta Goll. Sanfilippo and Riedel, 1973, p. 526, pl. 15, fig. 5; pl. 31, fig. 11. (This chapter: Pl. 2, Fig. 9.) Table 1, number 17; Tables 2, 3, number $13^{\prime}$.

Dendrospyris anthocyrtoides (Butschli). Riedel and Sanfilippo, 1971, p. 1590 , pl. 5, figs. 5-7. Table 1 , number 10 ; Tables 2 , 3 , number $6^{\prime}$.

Dendrospyris binapertonis Goll, 1968, p. 1420, pl. 173, figs. 5, 6, 10, 11; text-fig. 8. (This chapter: Pl. 3, Fig. 4.) Table 1, number 4; Tables 2, 3, number $10^{\prime}$.

Dendrospyris damaecornis (Haeckel). Goll, 1968, p. 1420, pl. 173, figs. 1-4; text-fig. 8. (This chapter: Pl. 3, Fig. 3.) Table 1, number 15; Tables 2, 3, number 16 '.

Dendrospyris fragoides Sanfilippo and Riedel, 1973, pl. 526, pl. 15, figs. 8-13; pl. 31 , figs. 13,14 . Table 1 , number 45 ; Tables 2,3 , number $32^{\prime}$.

Dendrospyris inferispina Goll, 1968, p. 1421, pl. 174, figs. 5-8, 10; text-fig. 8 . Table 1 , number 74 ; Tables 2,3 , number $70^{\prime}$.

Dendrospyris pannosa Goll, 1968, p. 1421, pl. 173, figs. 13, 15, 19; text-fig. 8. Table 1, number 59; Tables 2, 3, number $54^{\prime}$.

Dendrospyris pododendros (Carnevale). Goll, 1968, p. 1422, pl. 174, figs. 1-4; text-fig. 8. Table 1, number 34; Tables 2, 3, number $60^{\prime}$.

Dendrospyris stabilis Goll, 1968, p. 1422, pl. 173, figs. 16-18, 20; textfig. 8. (This chapter: Pl. 2, Fig. 4.) Table 1, number 16; Tables 2, 3 , number $15^{\prime}$.

Dendrospyris stylophora (Ehrenberg). Goll, 1968, p. 1423, pl. 173, figs. 21-24; text-fig. 8. Table 1, number 33 .

Dendrospyris turriturcica-dasyotus Sanfilippo and Riedel, 1973, p. 527 , pl. 16 , fig. 3 ; pl. 32 , fig. 3 . Table 1 , number 80 ; Tables 2,3 , number $69^{\prime}$.

Dendrospyris turriturcica-turriturcica Sanfilippo and Riedel, 1973, p. 527 , pl. 16 , fig. 1 ; pl. 31 , fig. 15 ; pl. 32 , fig. 1 . Table 1 , number 63 ; Tables 2, 3, number $56^{\prime}$.

Dictyoprora amphora (Haeckel) group. Nigrini, 1977, p. 250, pl. 4, figs. 1, 2. (This chapter: Pl. 4, Fig. 6.) Table 1, number 56; Tables 2,3 , number $46^{\prime}$. 
Dictyoprora armadillo (Ehrenberg). Nigrini, 1977, p. 250, pl. 4, fig. 4. (This chapter: Pl. 4, Fig. 5.) Table 1, number 76; Tables 2, 3, number $74^{\prime}$.

Dictyoprora mongolfieri (Ehrenberg). Nigrini, 1977, p. 250, pl. 4, fig. 7. (This chapter: Pl. 4, Figs. 8-10.) Table 1, number 50; Tables 2 , 3 , number $38^{\prime}$.

Dictyoprora ovata (Haeckel). Nigrini, 1977, p. 251, pl. 4, figs. 5, 6. Table 1, number 37; Tables 2, 3, number $58^{\prime}$.

Dictyoprora pirum (Ehrenberg). Nigrini, 1977, p. 251, pl. 4, fig. 8. (This chapter: Pl. 4, Fig. 7.) Table 1, number 72; Tables 2, 3, number $66^{\prime}$.

Dictyoprora urceolus (Haeckel). Nigrini, 1977, p. 251, pl. 4, figs. 9, 10. (This chapter: Pl. 4, Fig. 14.) Table 1, number 19; Tables 1, 2, number $18^{\prime}$.

Dictyospyris discus Sanfilippo and Riedel, 1973, p. 527, pl. 16, figs. 4-8; pl. 32, figs. 4-7. Table 1 , number 81 ; Tables 2,3 , number $76^{\prime}$.

Dictyospyris gigas Ehrenberg. Sanfilippo and Riedel, 1973, p. 527, pl. 16, figs. 9, 10; pl. 32, figs. 10, 11. Table 1, number 78; Tables 2,3 , number $72^{\prime}$.

Dorcadospyris alata (Riedel), Riedel and Sanfilippo, 1970, p. 523, pl. 14, fig. 5; 1971, pl. 2D, fig. 1; Moore, 1971, pl. 11, figs. 3, 4. Table 1 , number 47.

Dorcadospyris argisca (Ehrenberg). Goll, 1969, pl. 56, figs. 9-11; textfig. (This chapter: Pl. 2, Fig. 12.) Table 1, number 60; Tables 2, 3 , number $51^{\prime}$.

Dorcadospyris ateuchus (Ehrenberg). Riedel and Sanfilippo, 1970, p. 523 , pl. 15 , fig. $4 ; 1971$, p. 1590, pl. 2D, fig. 6; pl. 3A, figs. 9, 10. Table 1, number 43; Tables 2, 3, number $30^{\prime}$.

Dorcadospyris(?) or Petalospyris(?) ateuchus (Ehrenberg), Petrushevskaya and Kozlova, 1972, p. 532 (in Nigrini, 1977).

Dorcadospyris circulus (Haeckel). Moore, 1971, p. 739, pl. 8, figs. 3-5. (This chapter: Pl. 3, Fig. 9.) Table 1, number 70.

Dorcadospyris confluens (Ehrenberg). Goll, 1969, p. 337, pl. 58, figs. 9-12; Sanfilippo and Riedel, 1973, p. 528, pl. 17, figs. 6-10; pl. 33, fig. 1. (This chapter: Pl. 2, Fig. 8.) Table 1, number 18; Tables 2, 3 , number $20^{\prime}$

Dorcadospyris costatescens Goll, 1969, p. 337, pl. 57, figs. 1-4; textfig. 2. Table 1, number 44; Tables 2, 3, number 31'

Dorcadospyris dentata Haeckel, 1887, p. 1040, pl. 85, fig. 6. Riedel, 1957, p. 79, pl. 1, fig. 4. (This chapter; Pl. 3, Fig. 7.) Table 1, number 53; Tables 2,3 , number $41^{\prime}$.

Dorcadospyris forcipata (Haeckel). Riedel and Sanfilippo, 1970, p. 523, pl. 15, fig. 7; Moore, 1971, p. 740, pl. 10. (This chapter: Pl. 3, Fig. 8.) Table 1, number 42; Tables 2,3 , number $29^{\prime}$.

Dorcadospyris papilio (Riedel). Riedel and Sanfilippo, 1970, p. 523 , pl. 15 , fig. 5 ; Moore, 1971, p. 739 , pl. 8 , figs. 6, 7. Table 1 , number 67; Tables 2, 3, number 62'.

Dorcadospyris pentagona (Ehrenberg). Goll,, 1969, p. 338, pl. 59, figs. $8,10,12$; text-fig. 2 . (This chapter: Pl. 1, Fig. 9.) Table 1 , number 22; Tables 2, 3, number 21'.

Dorcadospyris platyacantha (Ehrenberg). Sanfilippo and Riedel, 1973 , p. 528 , pl. 17 , figs. $11-15$; pl. 33, fig. 2. Table 1 , number 68 ; Tables 2, 3, number $67^{\prime}$.

Dorcadospyris praeforcipata Moore, 1971, p. 738, pl. 9, figs. 4-7. Table 1, number 73; Tables 2, 3, number $65^{\prime}$.

Dorcadospyris pseudopapilio Moore, 1971 , p. 738 , pl. 6, figs. 7, 8 . (This chapter: Pl. 3, Fig. 6.) Table 1, number 71; Tables 2, 3, number $64^{\prime}$.

Dorcadospyris quadripes Moore, 1971, p. 738, pl. 7, figs. 3-5. Table 1 , number 75; Tables 2, 3 , number $63^{\prime}$.

Dorcadospyris riedeli Moore, 1971, p. 739, pl. 9, figs. 1-3. Table 1, number 79; Tables 2, 3, number 73'.

Dorcadospyris simplex (Riedel). Riedel and Sanfilippo, 1970, pl. 15. (This chapter: P1. 3, Fig. 5.) Table 1, number 65; Tables 2, 3, number $59^{\prime}$.

Dorcadospyris spinosa Moore, 1971, p. 739, pl. 7, figs. 1, 2. (This chapter: Pl. 3, Fig. 10.)

Giraffospyris angulata (Haeckel). Goll, 1969, p. 331, pl. 59, figs. 4, $6,7,9$; text-fig. 2. Table 1 , number 1 ; Tables 2,3 , number $3^{\prime}$.

Giraffospyris annulispina Goll, 1969, p. 331, pl. 57, figs. 11, 12, 15, 17; text-fig. 2. Table 1, number 23; Tables 2, 3, number 22 '
Giraffospyris circumflexa Goll, 1969, p. 332, pl. 60, figs. 1-4; text-fig. 2. (This chapter: Pl. 1, Figs. 1, 2.) Tables 2, 3, number 4'.

Giraffospyris cyrillium Sanfilippo and Riedel, 1973, p. 528, pl. 18, figs. 1-3; pl. 33, fig. 3. (This chapter: Pl. 2, Fig. 7.) Table 1, number 61; Tables 2, 3, number 52'.

Giraffospyris didiceros (Ehrenberg). Goll, 1969, p. 332, pl. 60, figs. 5-7, 9; text-fig. 2. (This chapter: Pl. 2, Fig. 13.) Table 1, number 32; Tables 2, 3, number $36^{\prime}$.

Giraffospyris haeckelii (Bütschli). Goll, 1969, p. 334, pl. 57, figs. 5-8; text-fig. 2. Table 1, number 57; Tables 2, 3, number 48'.

Giraffospyris lata Goll, 1969, p. 334, pl. 58, figs. 22, 24-26; text-fig. 2; Sanfilippo and Riedel, 1973, p. 529, pl. 18, figs. 3-7; pl. 33, fig. 4. (This chapter: Pl. 2, Fig. 5.) Table 1, number 77; Tables 2, 3, number $71^{\prime}$.

Giraffospyris laterispina Goll, 1969, p. 334, pl. 58, figs. 15, 16, 20, 21; text-fig. 2. Table 1, number 6; Tables 2, 3, number 12'.

Giraffospyris toxaria (Haeckel). Goll, 1969, p. 334, pl. 56, fig. 1, 2, 4, 7 ; text-fig. 2. Table 1 , number 35 .

Liriospyris clathrata (Ehrenberg). Goll, 1968, p. 1426, pl. 175, Figs. 12, 13, 16, 17; text-fig. 9. (This chapter: Pl. 1, Fig. 8.) Table 1, number 5; Tables 2, 3, number 11'.

Liriospyris elevata Goll, 1968, p. 1426, pl. 175, figs. 4, 5, 8, 9; text-fig. 9. Table 1, number 27; Tables 2,3 , number $34^{\prime}$.

Liriospyris geniculosa Goll, 1968, p. 1427, pl. 175, figs. 21-24; textfig. 9. (This chapter: Pl. 1, Fig. 6.) Table 1, number 8 .

Liriospyris mutuaria Goll, 1968, p. 1428, pl. 175, figs. 6, 10, 11, 14; text-fig. 9. (This chapter: Pl. 2, Fig. 1.) Table 1, number 7; Tables 2,3 , number 7 '.

Liriospyris ovalis Goll, 1968, p. 1429, pl. 176, figs. 4, 6, 7; text-fig. 9. (This chapter: Pl. 2, Fig. 3.)

Liriospyris parkerae Riedel and Sanfilippo, 1971, p. 1590, pl. 2C, fig. 15; pl. 5, fig. 4. (This chapter: Pl. 1, Fig. 11.) Table 1, number 52; Tables 2, 3, number $40^{\prime}$.

Liriospyris reticulata (Ehrenberg). Goll, 1968, p. 1429, pl. 176, figs. 9, 11, 13; text-fig. 9. Table 1, number 36 ; Tables 2 , 3 , number $26^{\prime}$.

Liriospyris spinulosa (Ehrenberg). Goll, 1968, p. 1430, pl. 176, figs. 15, 18-20; text-fig. 9. (This chapter: Pl. 1, Fig. 4.) Table 1, number 58; Tables 2, 3, number $68^{\prime}$.

Liriospyris stauropora (Haeckel). Goll, 1968, p. 1431, pl. 175, figs. 1-3, 7; text-fig. 9. (This chapter: Pl. 1, Figs. 12, 13.) Table 1, number 28; Tables 2, 3, number $27^{\prime}$.

Petalospyris triceros (Ehrenberg) group. Petrushevskaya and Kozlova, 1972, p. 532, pl. 40, fig. 9; Nigrini, 1974, p. 1066, pl. 2B, figs. 5, 6. Table 1 , number 39 ; Tables 2,3 , number $50^{\prime}$.

Phormostichoartus corbula (Harting). Nigrini, 1977, p. 252, pl. 1, fig. 10. Table 1, number 64; Tables 2,3 , number $57^{\prime}$.

Phormostichoartus doliolum (Riedel and Sanfilippo). Nigrini, 1977, p. 252 , pl. 1, fig. 14 . Table 1 , number 25 ; Tables 2,3 , number $23^{\prime}$.

Phormostichoartus fistula Nigrini, 1977, p. 253, pl. 1, figs. 11-13. Table 1, number 46; Tables 2, 3, number 33'.

Phormostichoartus marylandicus (Martin). Nigrini, 1977, p. 253, pl. 2, figs. 1-3. (This chapter: Pl. 4, Fig. 15.) Table 1, number 21; Tables 2, 3, number $17^{\prime}$.

Rhabdolithis ellida Sanfilippo and Riedel, 1973, p. 529, pl. 18, figs. $8-11$; pl. 33 , figs. 5-8. Table 1 , number 54 ; Tables 2 , 3 , number $42^{\prime}$.

Rhabdolithis pipa Ehrenberg, 1854, pl. 36, fig. 59; 1875, p. 159, pl. 1 , fig. 27. Sanfilippo and Riedel, 1973, p. 529, pl. 18, figs. 12-16; pl. 33 , figs. 9,10 . Table 1 , number 49 ; Tables 2,3 , number $45^{\prime}$.

Siphocampe acephala (Ehrenberg). Nigrini, 1977, p. 254, pl. 3, fig. 5. (This chapter: Pl. 4, Fig. 12.) Table 1, number 62; Tables 2, 3, number $55^{\prime}$.

Siphocampe arachnea (Ehrenberg) group. Nigrini, 1977, p. 255. pl. 3, figs. 7,8 . Table 1 , number 24 ; tables 2,3 , number $25^{\prime}$.

Siphocampe elizabethae (Clark and Campbell). Nigrini, 1977, p. 256, pl. 3, fig. 6. (This chapter: Pl. 4, Fig. 11.) Table 1, number 66; Tables 2, 3, number $61^{\prime}$.

Siphocampe lineata (Ehrenberg) group. Nigrini, 1977, p. 256, pl. 3. figs. 9, 10. (This chapter: Pl. 4, Fig. 13.) Table 1, number 20; Tables 2, 3, number 19'.

Siphocampe nodosaria (Haeckel). Nigrini, 1977, p. 256, pl. 3, fig. 11. Table 1, number 30; Tables 2, 3, number $47^{\prime}$. 
Table 2. Cenozoic radiolarians, Hole 462, in samples with less than $1 \%$ reworked specimens. (See text for explanation.)

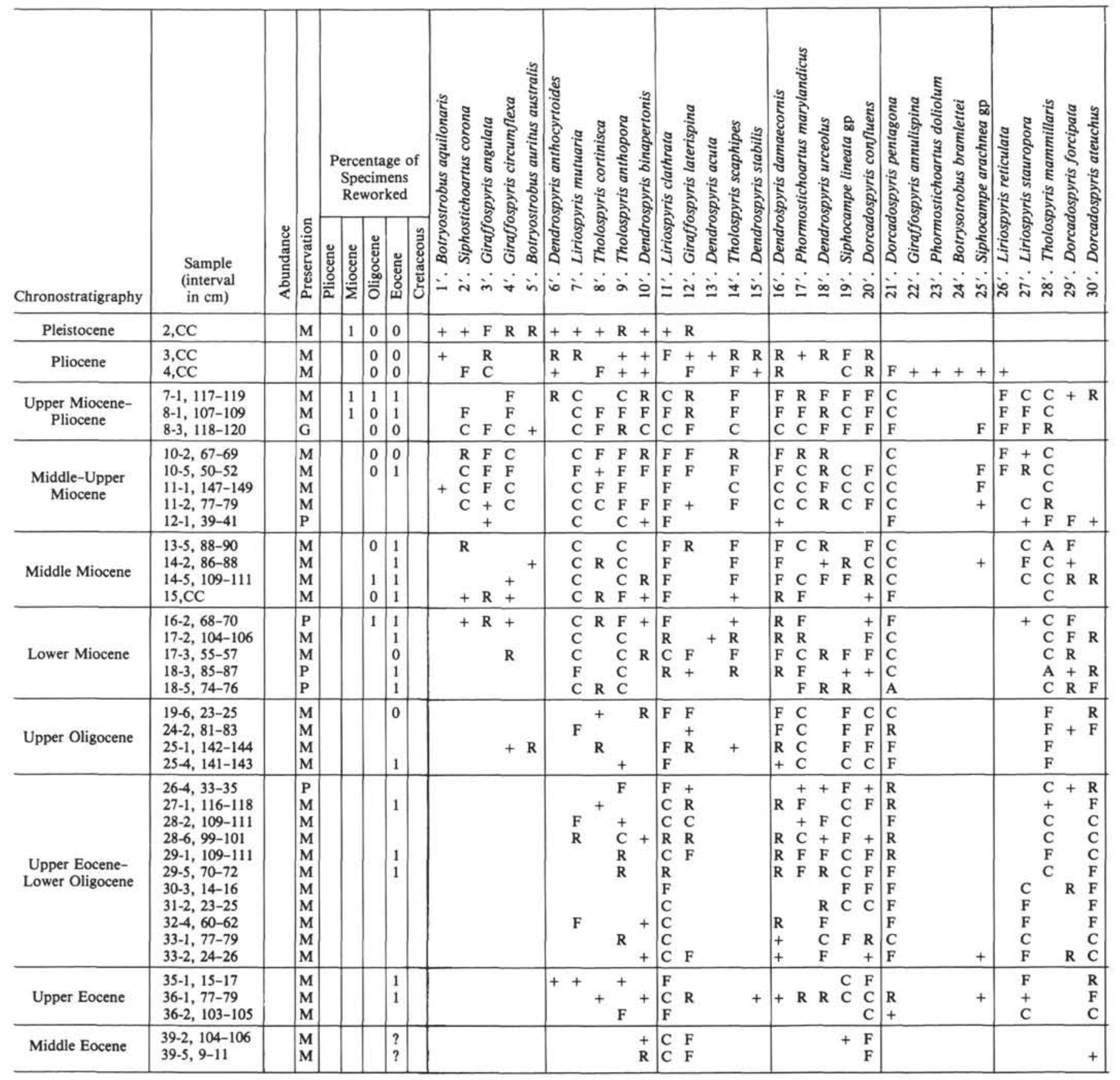

Siphocampe(?) quadrata (Petrushevskaya and Koslova). Nigrini, 1977 , p. 257 , pl. 3 , fig. 12 . Table 1 , number 69 ; Tables 2,3 , number $75^{\prime}$.

Siphostichoartus corona (Haeckel). Nigrini, 1977, p. 257, pl. 2, figs. 5, 6. (This chapter: Pl. 4, Fig. 3.) Table 1, number 11; Tables 2, 3, number 2.

Siphostichoartus praecorona Nigrini, 1977 , p. 258 , pl. 2, figs. $8,9$. (This chapter: Pl. 4, Fig. 4.) Table 1, number 41; Tables 2, 3, number $53^{\prime}$.

Spirocyrtis gyroscalaris Nigrini, 1977, p. 258, pl. 2, figs. 10, 11. Table 1, number 31 ; Tables 2,3 , number $35^{\prime}$.

Spirocyrtis subscalaris Nigrini, 1977, p. 259, pl. 3, figs. 1, 2. Table 1, number 38 ; Tables 2,3 , number $43^{\prime}$.

Spirocyrtis subtilis Petrushevskaya. Petrushevskaya and Kozlova, 1972 , p. 540 , pl. 24, figs. 22-24. Nigrini, 1977, p. 260, pl. 3 , fig. 3.
(This chapter: Pl. 4, Figs. 1, 2.) Table 1, number 55; Tables 2, 3, number $49^{\prime}$.

Tholospyris anthopora (Haeckel). Goll, 1969, p. 324, pl. 55, figs. 1-4; text-fig. 1. (This chapter: Pl. 1, Fig. 3.) Table 1, number 9; Tables 2,3 , number $9^{\prime}$.

Tholospyris cortinisca (Haeckel). Goll, 1969, p. 325, pl. 56, figs. 3, 5, 6 , 8; text-fig. 1. (This chapter: Pl. 3, Fig. 2.) Table 1, number 3; Tables 2, 3, number $8^{\prime}$.

Tholospyris kantiana (Haeckel). Goll, 1969, p. 327, pl. 58, figs. 17-19, 23; text-fig. 1. (This chapter: Pl. 2, Fig. 6.) Table 1, number 40; Tables 2, 3, number $37^{\prime}$.

Tholospyris mammillaris (Haeckel). Goll, 1969, p. 327, pl. 55, figs. 5, 6; text-fig. 1. (This chapter: Pl. 1, Fig. 5.) Table 1, number 29; Tables 2, 3, number $28^{\prime}$. 
Table 2. (Continued).

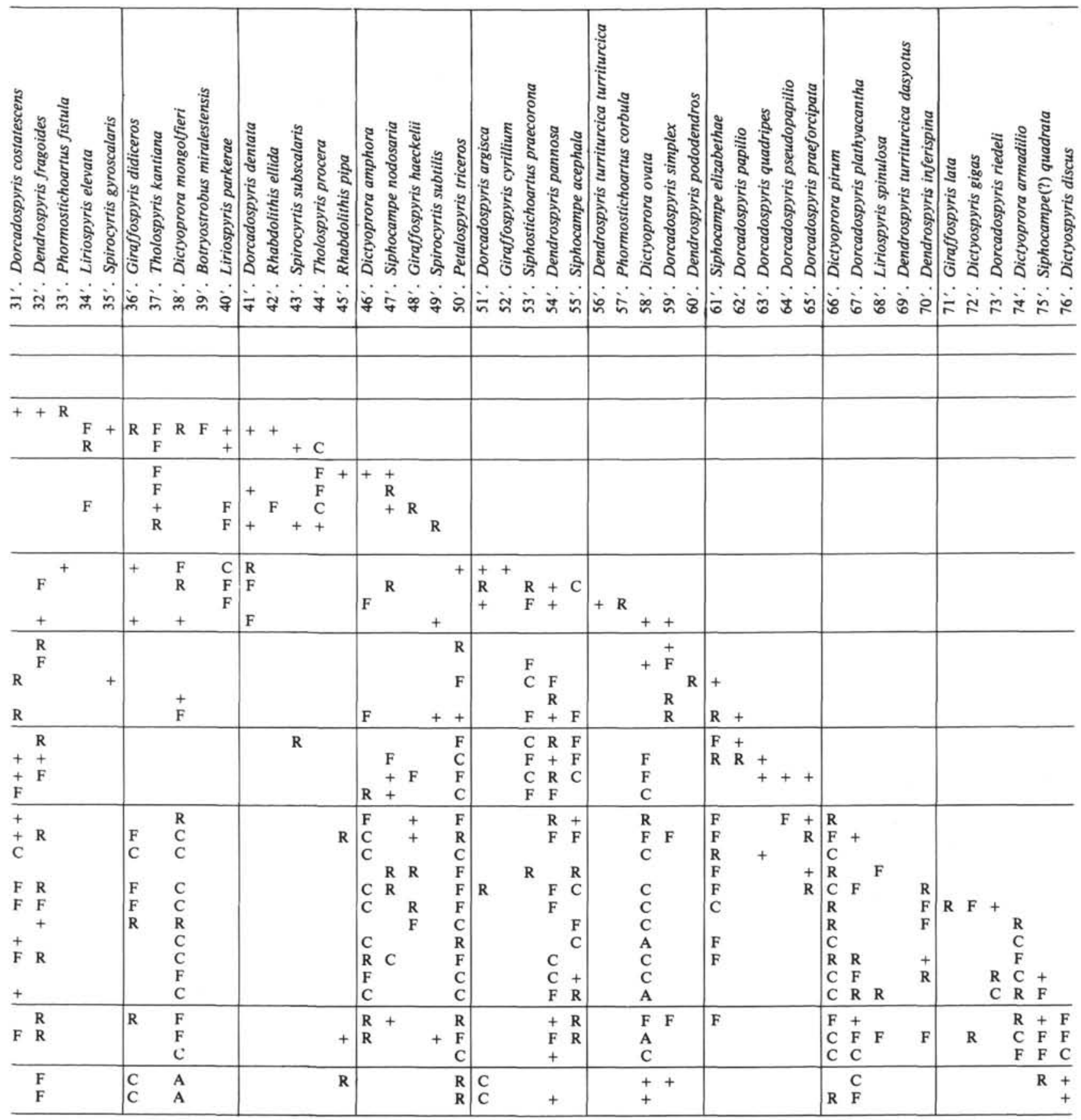

Tholospyris procera Goll, 1969, p. 328, pl. 59, figs. 8, 10-12; text-fig. 1. Table 1, number 48; Tables 2,3 , number $44^{\prime}$.

Tholospyris scaphipes (Haeckel). Goll, 1969, p. 328, pl. 58, figs. 1-8, 13, 14; text-fig. 1. (This chapter: Pl. 3, Fig. 1.) Table 1, number 14; Tables 2, 3, number $14^{\prime}$.

\section{MESOZOIC RADIOLARIANS}

Mesozoic radiolarians, even when common, were, except rarely, too poorly preserved to be useful for stratigraphic purposes. They were generally encountered in layers of claystone interbedded in eruptive rocks. It was therefore never possible to obtain a sequence of Meso- zoic radiolarians showing evolutionary changes. The original skeletons of the radiolarians have been replaced and filled with crystalline silica in the best cases, or recrystallized in calcite and/or zeolite, leaving only the gross external features preserved.

These poorly preserved forms cannot be identified to the species level, and seldom can be identified even to the genus level. Names cannot be used with great precision, and they are used conservatively without implying taxonomic agreement or disagreement. In order to provide bibliographic references to the species mentioned here, they are listed. When the published literature con- 
Table 3. Cenozoic radiolarians, Hole 462 , in samples with less than $1 \%$ reworked specimens. (See text for explanation.)

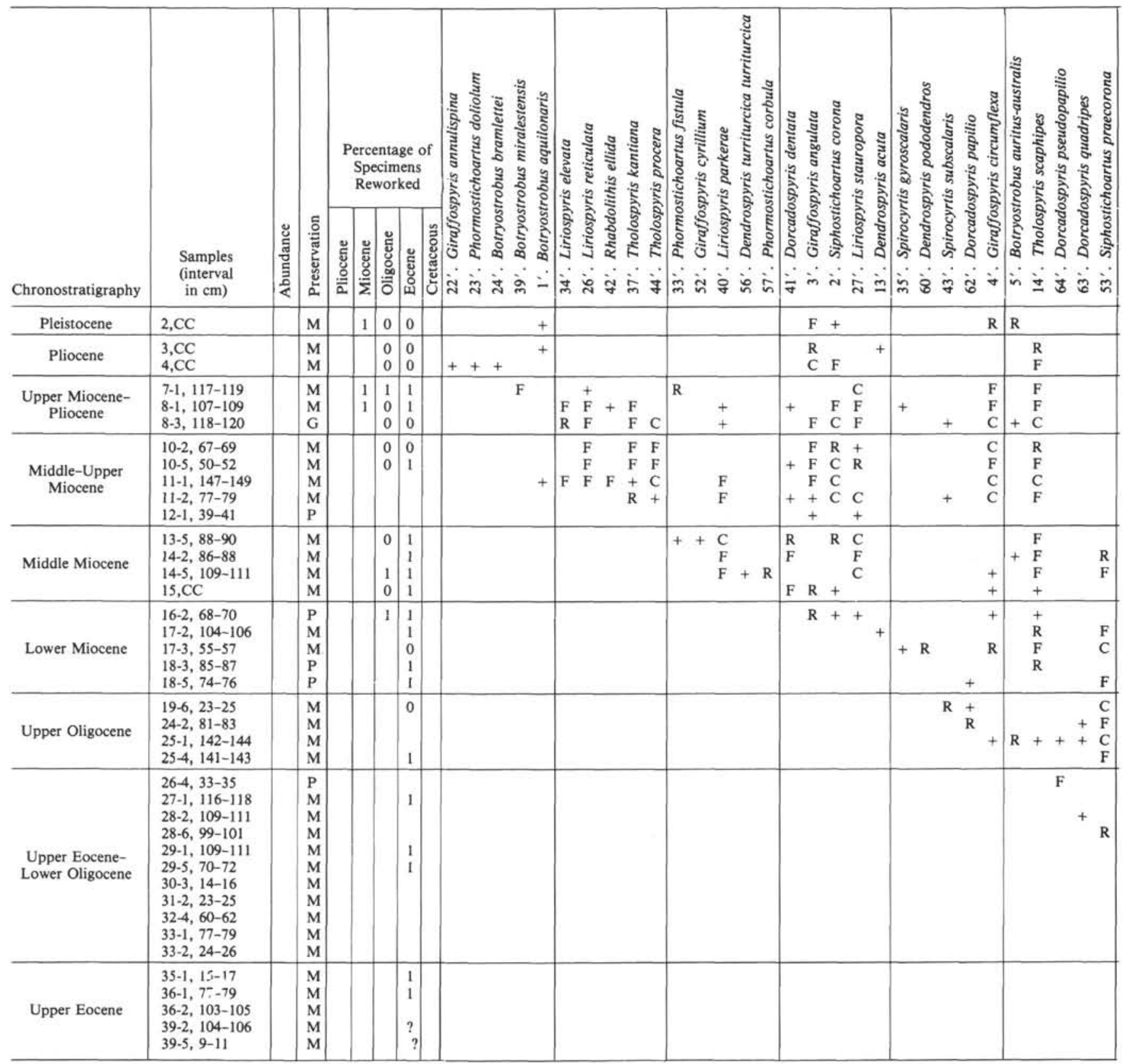

tains several different concepts of the limits of a species, the reference cited conforms to the concept as applied here.

In Hole 462, identification of radiolarians from the Cretaceous interval is nearly impossible because the tests have been replaced by zeolite and calcite, which crystallizes in large crystals that distort the specimens. However, Cores 51 (Section 3) and 55 (Section 4) provided somewhat better preserved specimens.

Similarly, in Hole 462A samples, as because of the poor preservation of the radiolarians (replaced by calcite and zeolite) and their distortion, only a few vague high-level taxa can be identified. In Core 43 (Sections 1 and 2), Core 46 (Section 1), and Core 80 (Section 1), however, some identifications were attempted.
The ages attributed to the samples are based on the works of Riedel and Sanfilippo (1974), Foreman (1975), and Pessagno (1977).

The samples yielding the best radiolarians are as follows:

Samples 462-51-3, 41-43 cm: Alievum gallowayi?, Dictyomitra torquata, Dictyomitra duedecimcostata, Dictyomitra lilyae, and Dictyomitra sp. aff. D. koslovae, suggest that this sample belongs to the Upper Cretaceous (Senonian).

Sample 462-55-4, 55-59 cm: Archaeospongoprunum sp., Alievum sp., Dictyomitra duodecimcostata, Dictyomitra lilyae, Dictyomitra somphedia, and Dictyomitra torquata: same as previous sample (Senonian).

Sample 462A-43-1, 36-40 cm: Dictyomitra lacrimula. 
Table 3. (Continued).

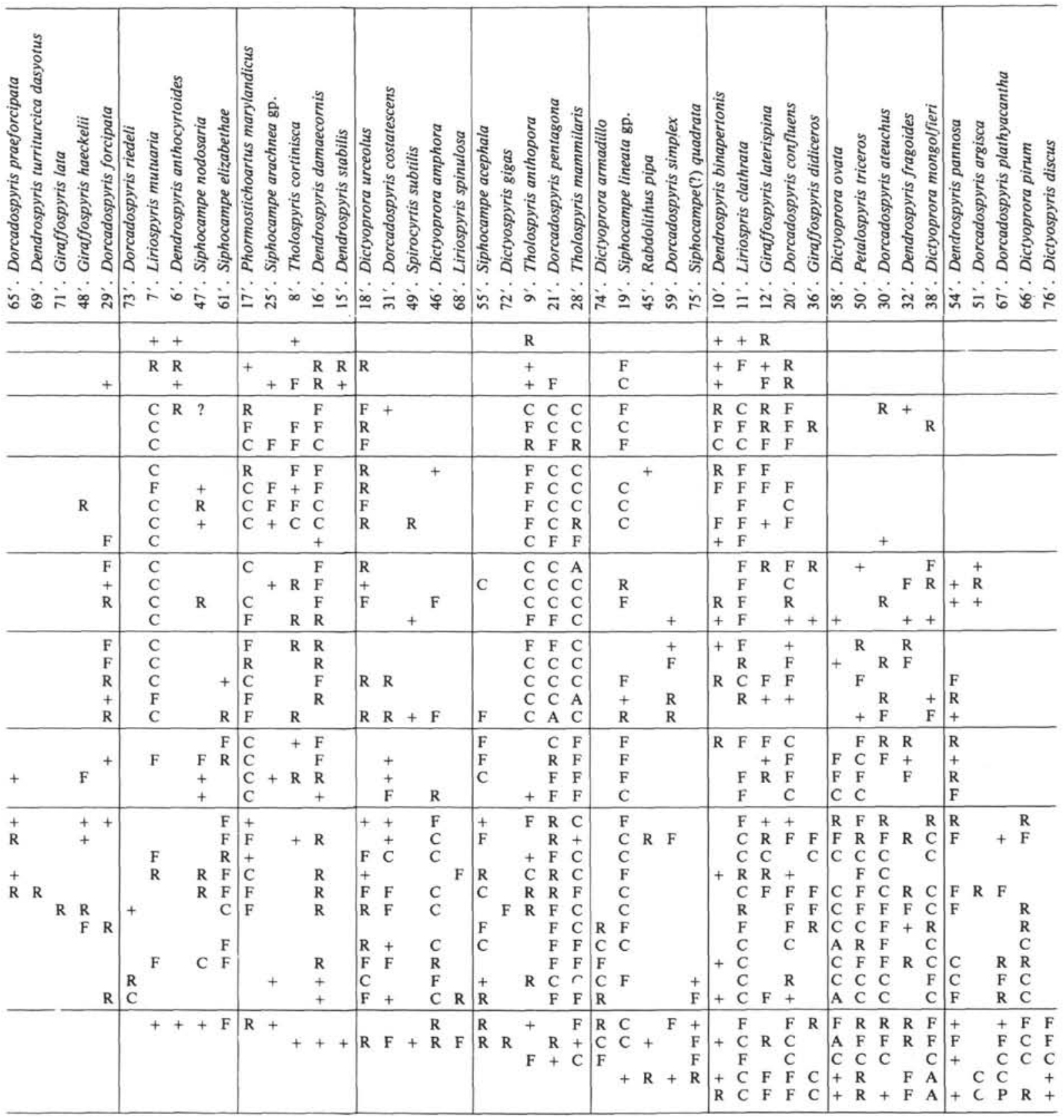

Sample 462A-43-2, 50-54 cm: Acanthocircus sp. (A. trizonalis?), Dictyomitra lacrimula, Emiluvia sp., Eucyrtis tenuis, Hagiastridae, Hemicryptocapsa spp. cf. $H$. capita, Mirifusus mediodilatatus, Thanarla elegantissima, Sethocapsa cetia?, Sethocapsa sp., spumellarians. These forms suggest the Eucyrtis tenuis Zone, which is Barremian to Albian p. p., according to Riedel and Sanfilippo (1975), and Valanginian to lower Aptian, according to Foreman, 1975. The specimens are probably Barremian.

Sample 462A-46-1, 1-3 cm: Acanthocircus carinatus?, Crucella sp., Dictyomitra lacrimula, Emiluvia pessagnoi?, Emiluvia sp. (E. chica?), Hagiastrum sp., Podobursa sp., spongosaturnalids, Staurosphaera se- decimporata. These radiolarians belong to the $E$. tenuis Zone, and, as for the previously mentioned sample, are probably Barremian.

Some radiolarians from the E. tenuis Zone again occur in the red-brown claystone interval (Sample 462A$80-1,16-17 \mathrm{~cm}$ ), and suggest the same age. The following radiolarians were recognized: Crucella sp., Dictyomitra lacrimula, Eucyrtis micropora(?), Sethocapsa sp., Thanarla elegantissima, Thanarla sp. aff. T. conica, and Mirifusus mediodilatatus.

\section{LIST OF MESOZOIC SPECIES}

Acanthocircus carinatus Foreman, 1975, p. 610, pl. 2C, fig. 8; pl. 4, fig. 12. 
Acanthocircus trizonalis (Rüst). Foreman, 1975, p. 610, pl. 20, figs. $1-4$.

Alievum gallowayi (White). Pessagno, 1963, p. 202, pl. 2, figs. 1, 3, 6; pl. 4 , figs. $2,5,7$; pl. 7, figs. 2,4 .

Dictyomitra duodecimcostata (Squinabol). Foreman, 1975, p. 614, pl. $1 G$, figs. 5, 6; pl. 7, fig. 11; pl. 16, fig. 3 .

Dictyomitra koslovae Foreman, 1975, p. 614, pl. 7, fig. 4.

Dictyomitra lacrimula Foreman, 1973b, p. 263, pl. 10, fig. 11.

Dictyomitra lilyae Tan Sin Hok, 1927, p. 55, pl. 10, fig. 83.

Dictyomitra somphedia Foreman, 1975, p. 614, pl. 7, figs. 11-13.

Dictyomitra torquata Foreman, 1971, p. 1676, pl. 3, fig. 4.

Emiluvia chica Foreman s. 1., Foreman, 1978, p. 744, pl. 1, fig. 4.

Emiluvia pessagnoi Foreman, 1973b, p. 262, pl. 8, fig. 6.

Eucyrtis micropora (Squinabol), Foreman, 1975, p. 615, pl. 2I, figs. 2-5.

Eucyrtis tenuis (Rüst), Foreman, 1975, p. 615, pl. 2I, figs. 7-9.

Hemicryptocapsa spp. cf. H. capita Tan Sin Hok, Foreman, 1975, pp. 579-676, pl. 2I, figs. $18,20$.

Mirifusus mediodilatata (Rüst), Foreman, 1978, p. 746, pl. 2, fig. 3.

Sethocapsa cetia Foreman, 1973b, p. 267, pl. 12, fig. 1; pl. 16, fig. 19.

Spongosaturnalis hueyi (Pessagno), Foreman, 1975, pl. 1A, fig. 6; pl. 4 , fig. 10.

Staurosphaera sedecimporata Rüst, 1885, p. 288, pl. XXVIII (III), fig. 1.

Thanarla elegantissima (Cita), Pessagno, 1977, p. 46, pl. 7, fig. 10.

Thanarla sp. aff. T. conica (Aliev), Pessagno, 1977, p. 46, pl. 7, figs. 8-14.

\section{ACKNOWLEDGMENTS}

I thank the Deep Sea Drilling Project curatorial staff at Scripps Institution of Oceanography (La Jolla, California) for supplying supplementary samples, and A. Schaaf, who reviewed the manuscript. Special thanks are due to W. R. Riedel, who, as usual, kindly offered useful discussions and assistance, and reviewed the manuscript.

Financial support for this investigation was provided in part by C.N.R.S. (France)-A.T.P. 3236.

\section{REFERENCES}

Ehrenberg, C. G., 1873. Grossere Felsproben des Polycysten-Mergels von Barbados mit weiteren Erlauterungen. Monatsber. Kgl. Preuss. Adad. Wiss. Berlin., Jahrg. 1873, pp. 213-263.

Foreman, H. P., 1971. Cretaceous Radiolaria. In Winterer, E. L., Riedel, W. R., et al., Init. Repts. DSDP, 7, Pt. 2: Washington (U.S. Govt. Printing Office), 1673-1693.

1973. Radiolaria from DSDP Leg 21. In Heezen, B. C., MacGregor, I. D., et al., Init. Repts. DSDP, 20: Washington (U.S. Govt. Printing Office), 249-305.

1975. Radiolaria from the North Pacific, Deep Sea Drilling Project, Leg 32. In Larson, R. L., Moberly, R., et al., Init. Repts. DSDP, 32: Washington (U.S. Govt. Printing Office), 579-676. ,1978. Mesozoic Radiolaria in the Atlantic Ocean off the Northwest coast of Africa, Deep Sea Drilling Project, Leg 41. In Lancelot, Y., Seibold, E., et al., Init. Repts. DSDP, 41: Washington (U.S. Govt. Printing Office), 739-760.
Goll, R. M., 1968. Classification and Phylogeny of Cenozoic Trissocyclidae (Radiolaria) in the Pacific and Caribbean basins. Part I. J. Paleontol., 43, no. 6:1409-1432.

1969. Classification and Phylogeny of Cenozoic Trissocyclidae (Radiolaria) in the Pacific and Caribbean basins. Part II. J. Paleontol. 42, no. 2:322-339.

Haeckel, E., 1887. Report on the Radiolaria collected by H.M.S. Challenger during the years 1873-76. Rept. Voyage Challenger, Zool., v. 18.

Moore, T. C., Jr., 1971. Radiolaria. In Tracey, J. I., Sutton, G. H., et al., Init. Repts. DSDP, 8: Washington (U.S. Govt. Printing Office), 727-775.

Nigrini, C., 1974. Cenozoic Radiolaria from the Arabian Sea, DSDP Leg 23. In Whitmarsh, R. B., Weser, O. E., Ross, D. A., et al., Init. Repts. DSDP, 23: Washington (U.S. Govt. Printing Office), 1051-1121.

, 1977. Tropical Cenozoic Artostrobiidae (Radiolaria). Micropaleontology, 23, no. 3:241-269.

Pessagno, E. A., Jr, 1963. Upper Cretaceous Radiolaria from Puerto Rico. Micropaleontology, 9, no. 2:197-214.

1977. Lower Cretaceous Radiolarian Biostratigraphy of the Great Valley Sequence and Franciscan Complex, California Coast Ranges. Cushman Foundation for Foraminiferal Research, Spec. Publ. no. 15, pp. 5-87.

Petrushevskaya, M. G., and Kozlova, G. E., 1972. Radiolaria: Leg 14, Deep Sea Drilling Project. In Hayes, D. E., Pimm, A. C., et al., Init. Repts. DSDP, 14: Washington (U.S. Govt. Printing Office), 495-648.

Riedel, W. R., 1957. Radiolaria: a preliminary stratigraphy. Rep. Swed. Deep-Sea Exped., 6, no. 3:59-96.

Riedel, W. R., and Sanfilippo, A., 1970. Radiolaria, Leg 4, Deep Sea Drilling Project. In Bader, R. G., Gerard, R. D., et al., Init. Repts. DSDP, 4: Washington (U.S. Govt. Printing Office), 503-575.

, 1971. Cenozoic Radiolaria from the western tropical Pacific, Leg 7. In Winterer, E. L., Riedel, W. R., et al., Init. Repts. DSDP, 7, Pt. 2: Washington (U.S. Govt. Printing Office), 1529-1672.

1974. Radiolaria from the Southern Indian Ocean. DSDP, Leg 26. In Davies, T. A., Luyendyk, B. P., et al., Init. Repts. DSDP, 26: Washington (U.S. Govt. Printing Office), 771813.

Rüst, D., 1885. Beiträge zur Kenntniss der fossilen Radiolarien aus Gesteinen des Jura. Palaeontographica, 31, ser. 3 (vol. 7): 273-321.

Sanfilippo, A., and Riedel, W. R., 1973. Cenozoic Radiolaria (exclusive of theoperids, artostrobiids and amphipyndacids) from the Gulf of Mexico, DSDP Leg 10. In Worzel, J. L., Bryant, W., et al., Init. Repts. DSDP, 10: Washington (U.S. Govt. Printing Office), 475-611.

Tan Sin Hok, 1927. Over de samenstelling en het ontstaan van krijt en mergel-gesteenten von de Molukken. In Brouwer, H. A., (Ed.), Geologische onderzoekingen in den oostelijken Oost-Indischen Archipel. V. Jaarb. Mijnwez. Ned. Oost-Indië, 55 (1926), Part 3:3-165. 

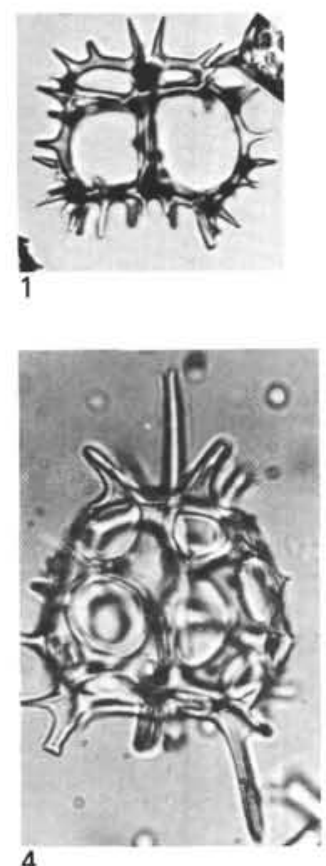

4

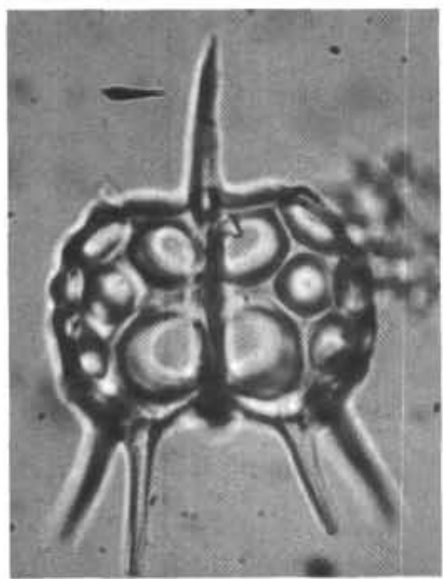

7

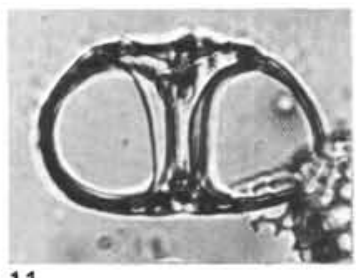

11

Plate 1. Cenozoic radiolarians, Hole 462.

Figures 1, 2. Giraffospyris circumflexa Goll. Fig. 1. Sample 462-7-3, $119-121 \mathrm{~cm}$. L $42 / 4 \times 200$. Fig. 2. Sample $462-10-2,67-69 \mathrm{~cm}$. F $21 / 3 \times 400$.

Figure 3. Tholospyris anthopora (Haeckel). Sample 462-7-3, 119-121 cm. M $27 / 4 \times 400$.

Figure 4. Liriospyris spinulosa (Ehrenberg). Sample 462-30-3, 14-16 $\mathrm{cm}$. E $15 / 4 \times 400$.

Figure 5. Tholospyris mammillaris (Haeckel). Sample 462A-2,CC $\times$ 260.

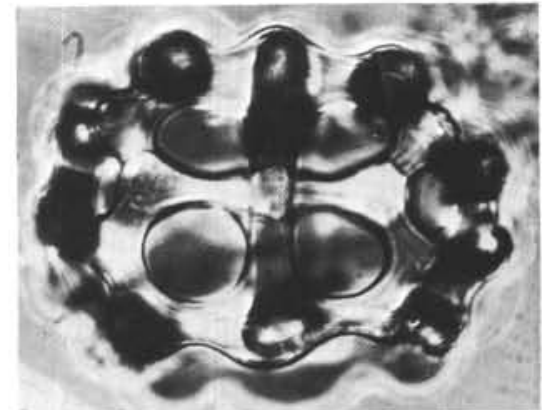

3

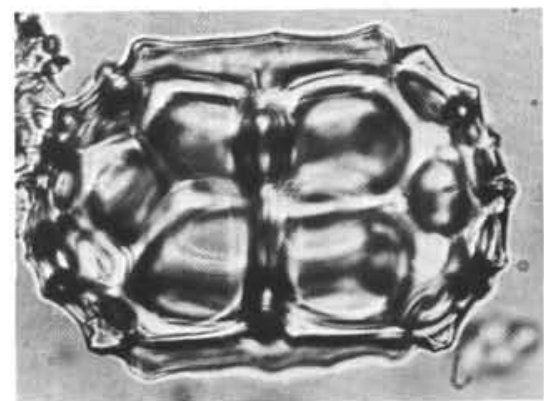

6

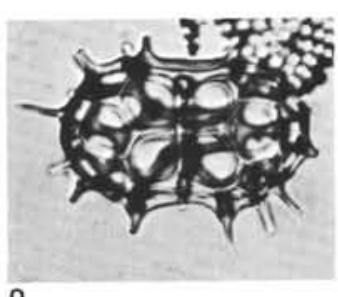

9

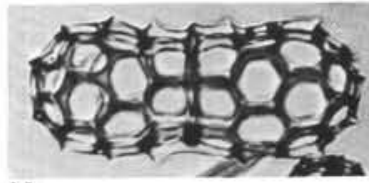

10

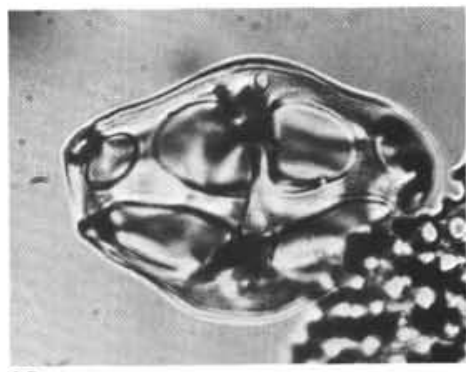

13

Figure 6. Liriospyris geniculosa Goll. Sample 462-7-3, 119-121 cm. R $18 / 2 \times 400$.

Figure 7. Spyrid sp. Sample 462-15,CC. D 10/0 × 420

Figure 8. Liriospyris clathrata (Ehrenberg). Sample 462-7-1, 117-119 cm. O $23 / 0 \times 400$.

Figure 9. Dorcadospyris pentagona (Ehrenberg). Sample 462-11-2, $77-79 \mathrm{~cm}$. P $14 / 4 \times 200$.

Figure 10. Spyrid. Sample 462-7-3, 119-121 cm. O 20/0 × 200 .

Figure 11. Liriospyris parkerae Riedel and Sanfilippo. Sample $462-11-1,147-149 \mathrm{~cm}$. R $17 / 0 \times 400$.

Figures 12, 13. Liriospyris stauropora (Haeckel). Fig. 1. Sample $462-$ 7-3, 119-121 cm. P 17/2 × 400. Fig. 2. Sample 462-7-3, 119-121 cm. P $17 / 3 \times 400$. 

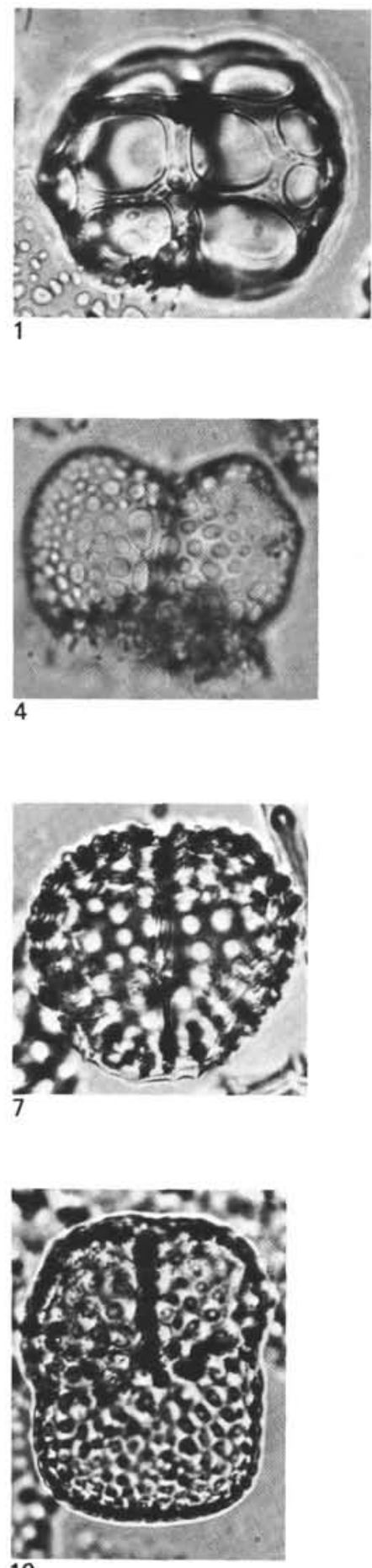

10

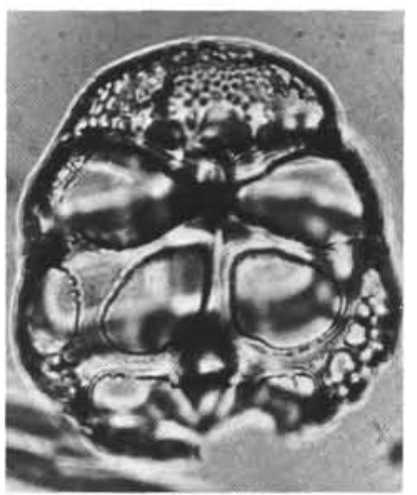

2

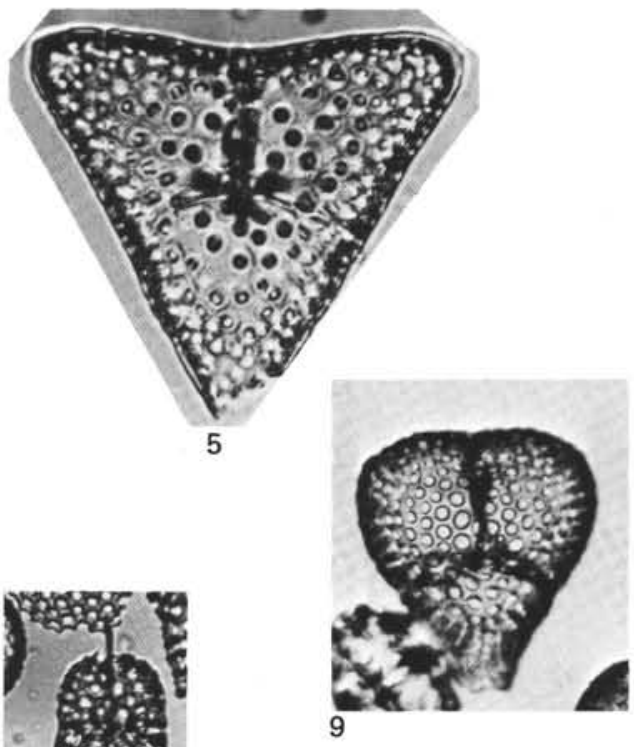

8

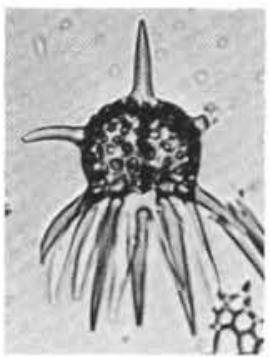

11

Plate 2. Cenozoic radiolarians, Hole 462.

Figure 1. Liriospyris mutuaria Goll. Sample 462-7-3, 119-121 cm. P $18 / 0 \times 400$.

Figure 2. Liriospyris sp. aff. L. reticulata. Sample 462-7-1, 117-119 cm. K $25 / 0 \times 400$.

Figure 3. Liriospyris ovalis. Sample 462-11-1, 147-149 cm. G 19/1 $\times$ 400.

Figure 4. Dendrospyris stabilis Goll. Sample 462-2,CC. $\times 420$.

Figure 5. Giraffospyris lata Goll. Sample 462-23-4, 67-69 cm. L 39/0 $\times 400$.

Figure 6. Tholospyris kantiana (Haeckel). Sample 462-7-3, 119-121 cm. K $22 / 1 \times 400$.

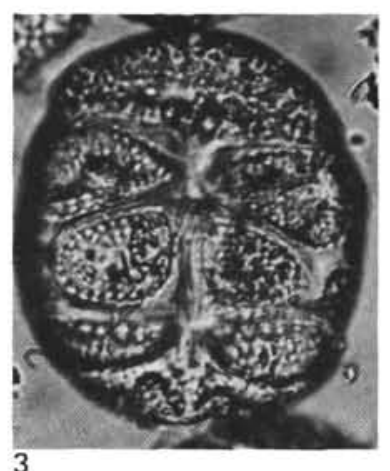

3
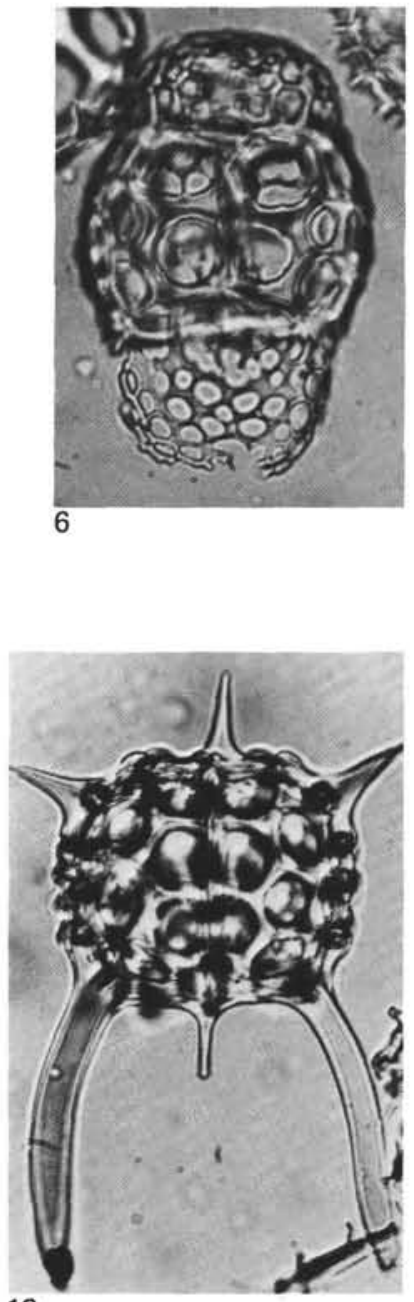

13

Figure 7. Giraffospyris cyrillium Sanfilippo and Riedel. Sample $462-29-5,70-72 \mathrm{~cm}$. U $17 / 4 \times 400$.

Figure 8. Dorcadospyris confluens (Ehrenberg). Sample 462-30-3, $14-16 \mathrm{~cm} . \mathrm{R} 19 / 0 \times 200$.

Figure 9. Dendrospyris acuta Goll. Sample 462-13-5, 88-90 cm. T $21 / 0 \times 200$.

Figure 10. Dendrospyris sp. Sample 462-3,CC. D $17 / 2 \times 400$.

Figure 11. Spyrid. Sample 462-30-3, 14-16 cm. K 19/1 × 200 .

Figure 12. Dorcadospyris argisca (Ehrenberg). Sample 462-39-5, 9-11 cm. J $17 / 2 \times 200$.

Figure 13. Giraffospyris didiceros (Ehrenberg). Sample 462-29-5, $70-72 \mathrm{~cm}$. P $20 / 0 \times 400$. 


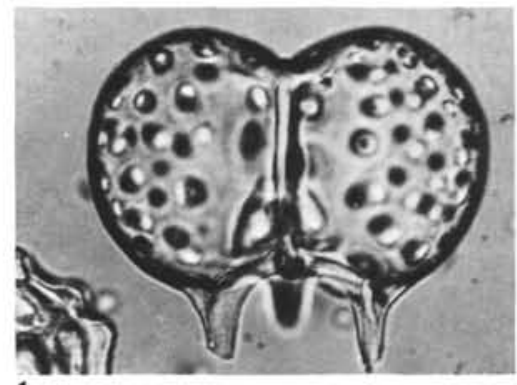

1

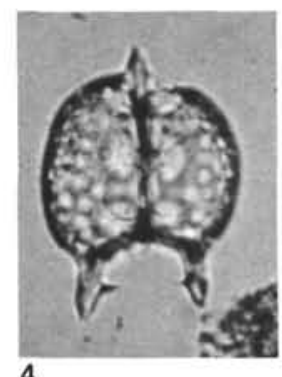

4
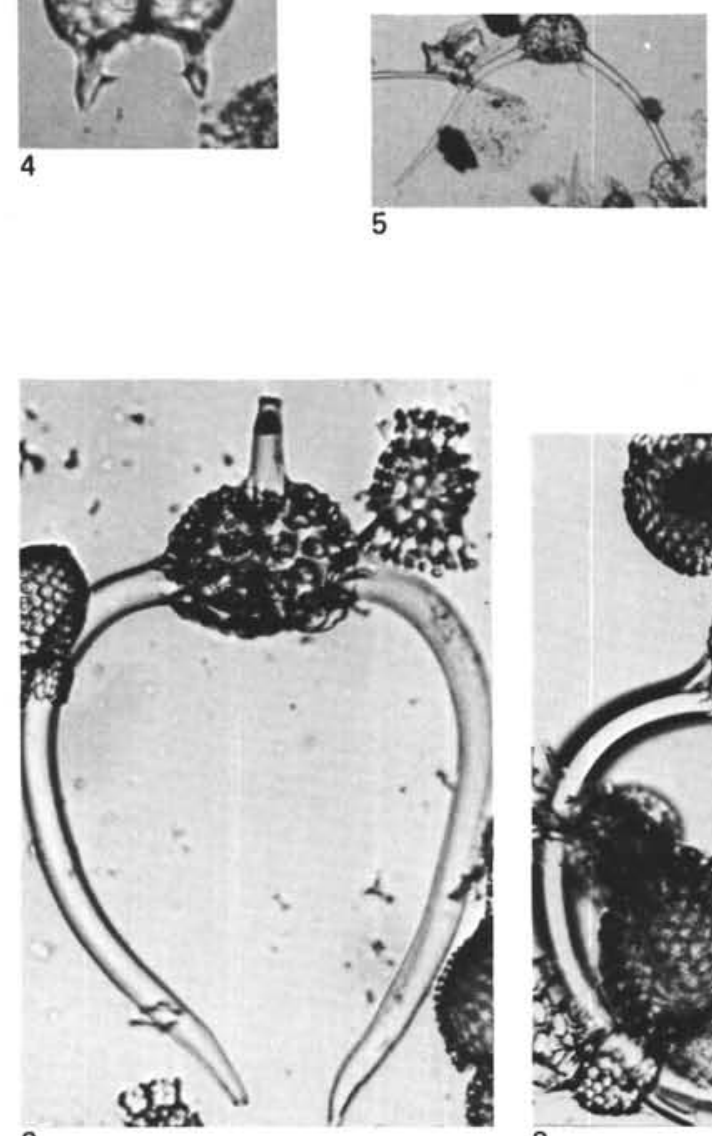

8

Plate 3. Cenozoic radiolarians, Hole 462.

Figure 1. Tholospyris scaphipes (Haeckel). Sample 462-7-3, 119-121 cm. L 20/1 $\times 400$.

Figure 2. Tholospyris cortinisca (Haeckel). Sample 462-15,CC. B 33/1 $\times 420$.

Figure 3. Dendrospyris damaecornis (Haeckel). Sample 462-7-3, $119-121 \mathrm{~cm}$. D $20 / 0 \times 400$.

Figure 4. Dendrospyris binapertonis Goll. Sample 462-2,CC. $\times 260$. Figure 5. Dorcadospyris simplex (Riedel). Sample 462-2,CC. $\times 82$.
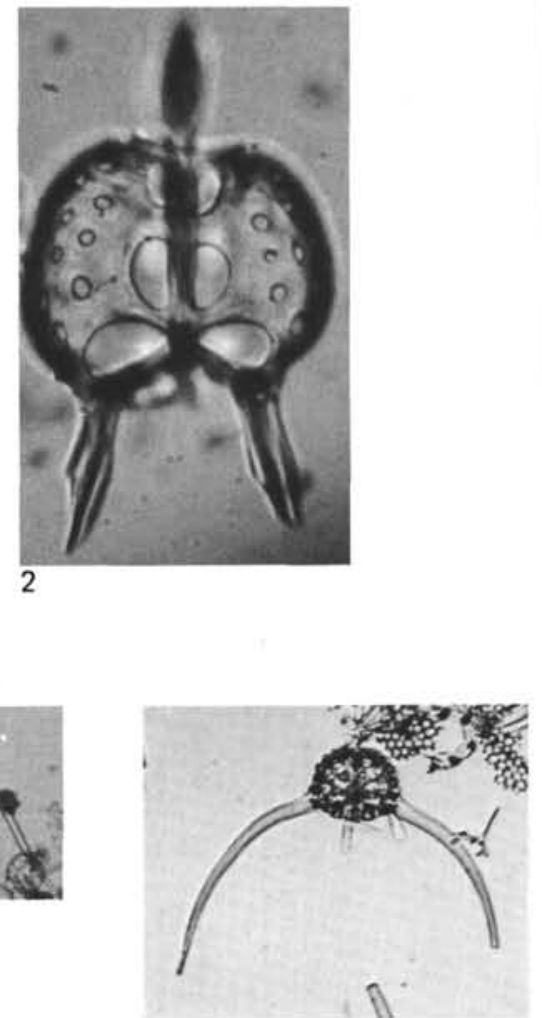

6
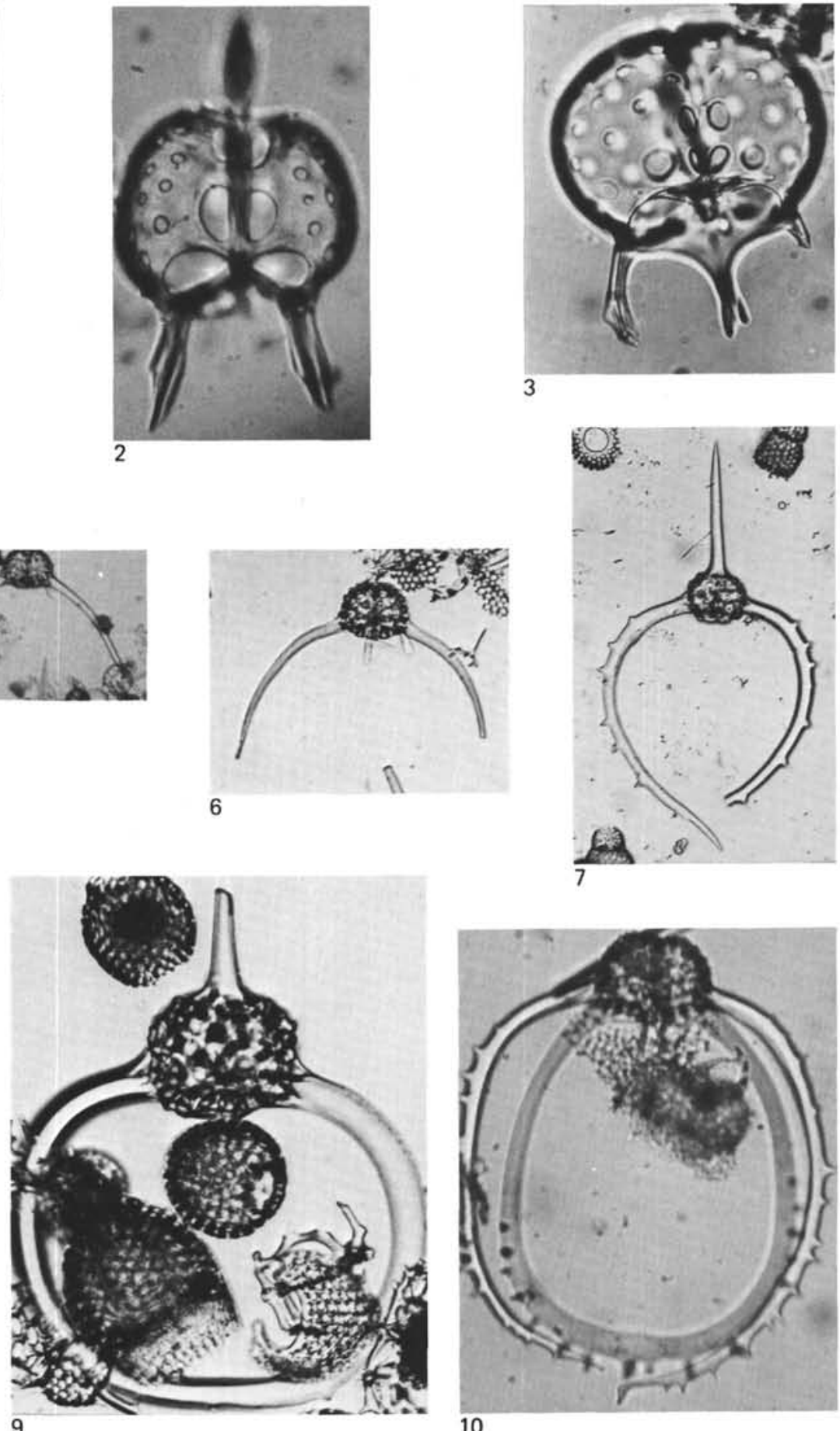

Figure 6. Dorcadospyris pseudopapilio Moore. Sample 462-30-3, 14-16 cm. P $17 / 4 \times 100$.

Figure 7. Dorcadospyris dentata Haeckel. Sample 462-15,CC. U 16/4 $\times 100$.

Figure 8. Dorcadospyris forcipata (Haeckel). Sample 462-13-5, 88-90 cm. F $21 / 2 \times 200$.

Figure 9. Dorcadospyris circulus (Haeckel). Sample 462-22-3, 106-108 cm. J $22 / 0 \times 200$.

Figure 10. Dorcadospyris spinosa Moore. Sample 462-23,CC. $\times 160$. 

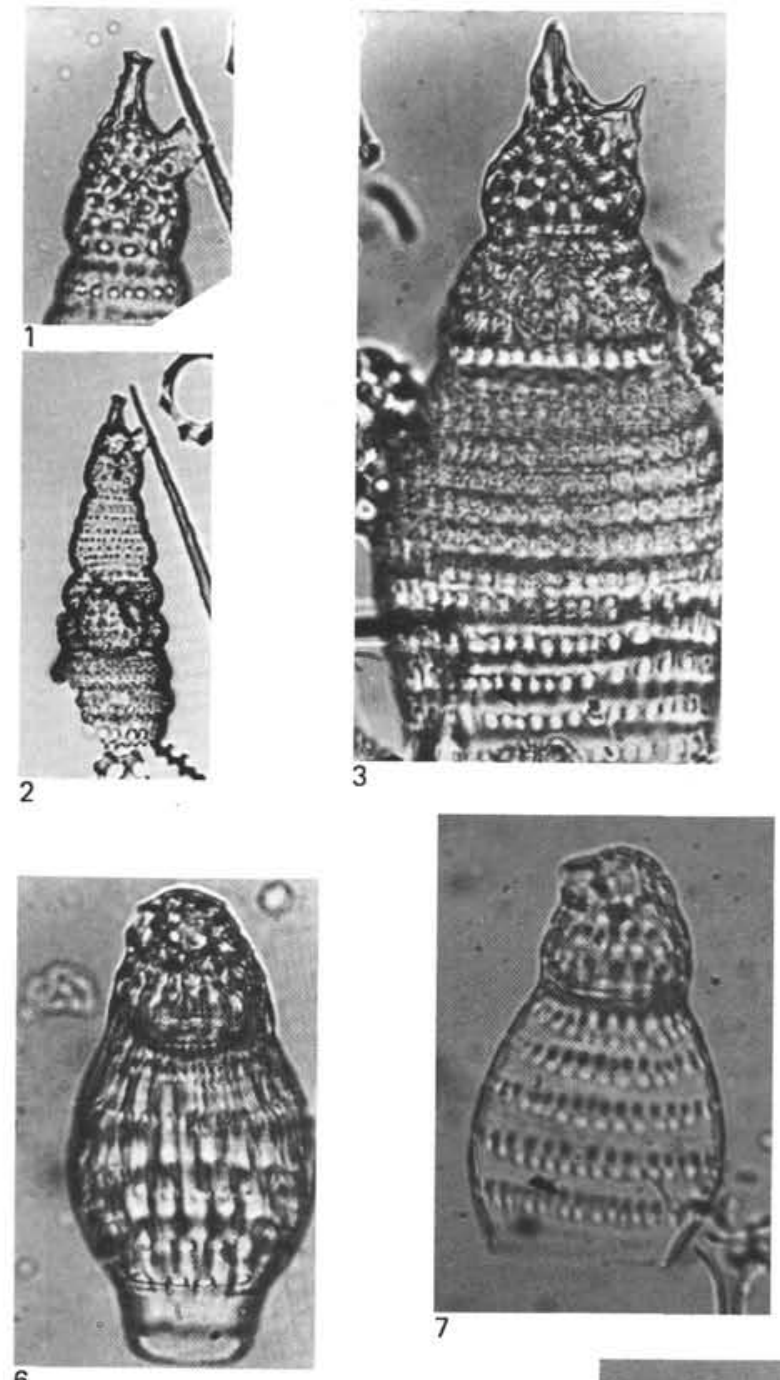

6
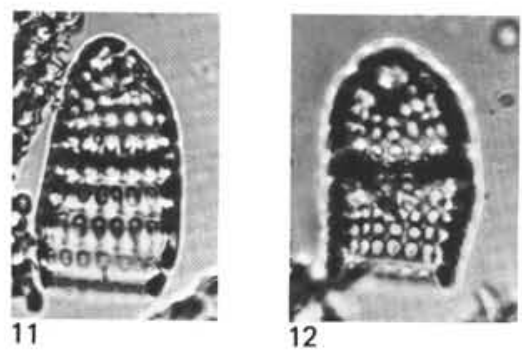

Plate 4. Cenozoic radiolarians, Hole 462.

Figure 1. Spirocyrtis subtilis Petrushevskaya. Sample 462-18-5, 74-76 $\mathrm{cm}$. V $23 / 1 \times 400$.

Figure 2. Spirocyrtis subtilis Petrushevskaya. Sample 462-18-5, 74-76 $\mathrm{cm}$. V $23 / 1 \times 200$

Figure 3. Siphostichoartus corona (Haeckel). Sample 462-10-5, 50-52 $\mathrm{cm}$. O $19 / 4 \times 400$.

Figure 4. Siphostichoartus praecorona Nigrini. Sample 462-17-3, 55$57 \mathrm{~cm}$. Q $19 / 3 \times 400$.

Figure 5. Dictyoprora armadillo (Ehrenberg). Sample 462-30-3, 14 $16 \mathrm{~cm}$. D $19 / 0 \times 200$.

Figure 6. Dictyoprora amphora group (Haeckel). Sample 462-3,CC. D $17 / 2 \times 400$.

Figure 7. Dictyoprora pirum (Ehrenberg). Sample 462A-2,CC. P 10/3 $\times 420$.
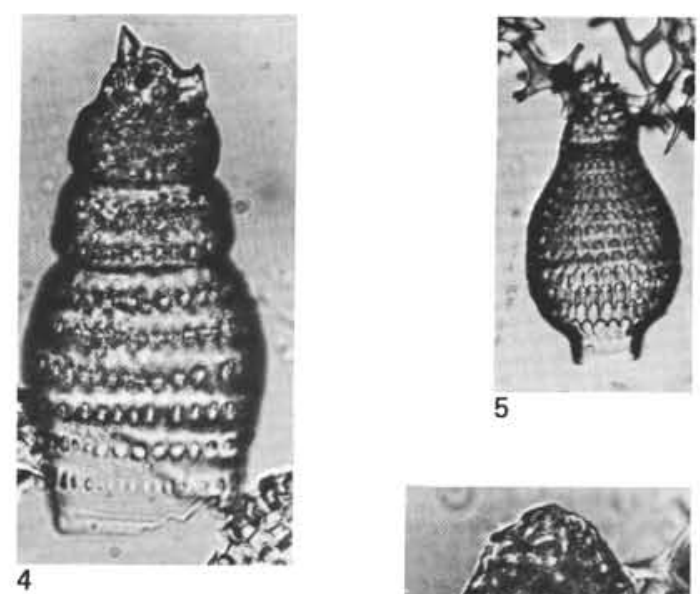

5
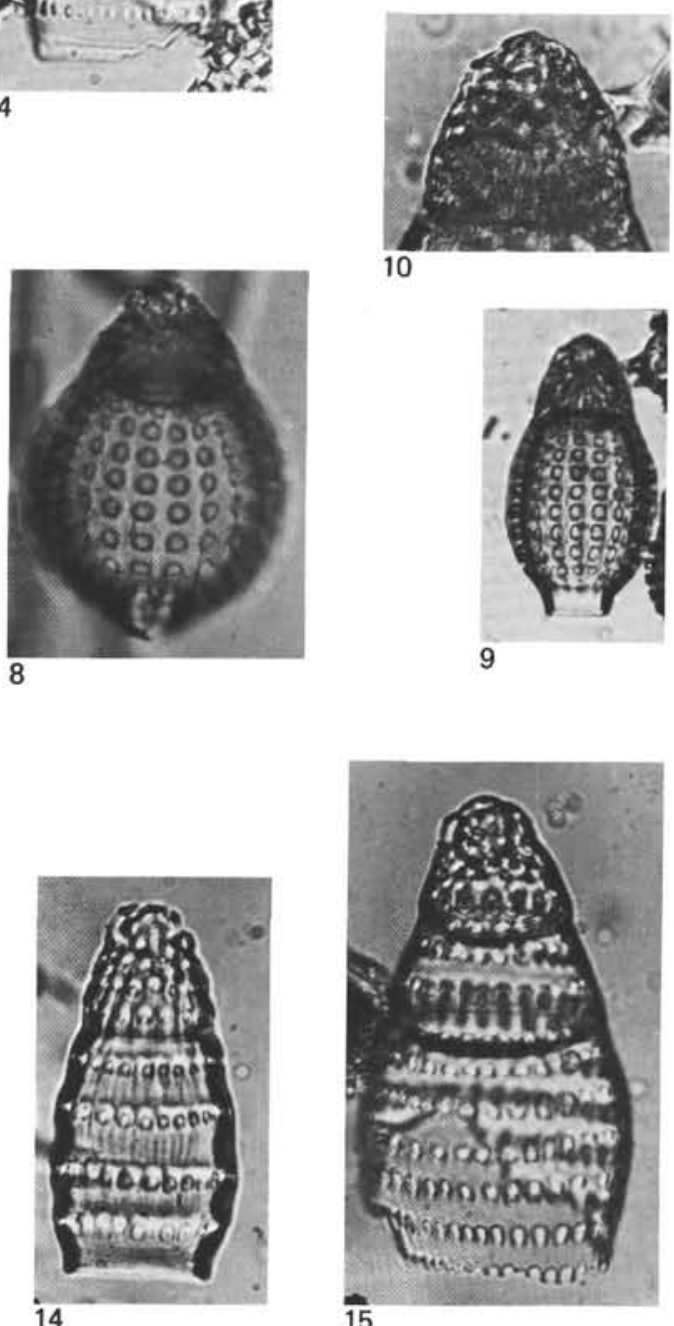

Figure 8. Dictyoprora mongolfieri (Ehrenberg) group. Sample 462A2, CC. L $12 / 4 \times 260$.

Figure 9. Dictyoprora mongolfieri (Ehrenberg) group. Sample 462$30-3,14-16 \mathrm{~cm}$. D $19 / 0 \times 200$.

Figure 10. Dictyoprora mongolfieri (Ehrenberg) group. Sample 462 30-3, 14-16 cm. D 19/0 × 400 .

Figure 11. Siphocampe elizabethae (Clark and Campbell). Sample $462-25-1,142-144 \mathrm{~cm}$. G 19/0 × 400 .

Figure 12. Siphocampe acephala (Ehrenberg). Sample 462-25-1, 142$144 \mathrm{~cm}$. G $19 / 1 \times 400$.

Figure 13. Siphocampe lineata (Ehrenberg) group. Sample 462-7-1, $117-119 \mathrm{~cm}$. S $23 / 0 \times 400$.

Figure 14. Dictyoprora urceolus (Haeckel). Sample 462-7-3, 119-121 $\mathrm{cm}$. L $42 / 0 \times 400$.

Figure 15. Phormostichoartus marylandicus (Martin). Sample 462$7-1,117-119 \mathrm{~cm}$. H $23 / 0 \times 400$. 